\title{
Quantifying the Accuracy of Hammerstein Model Estimation
}

\author{
Brett Ninness* $\quad$ Stuart Gibson $^{\dagger}$
}

\begin{abstract}
This paper investigates the accuracy of the linear component that forms part of an overall Hammerstein model-structure estimate, and a key finding is that the process of estimating the non-linear element can have a strong effect on the associated estimate of the linear dynamics. Furthermore, this effect is not explained simply by way of considering how the input spectrum is changed by the non-linearity. Instead, it arises that the linear model-estimate variability may be dominated by a term that depends on the frequency response of the linear system itself. Amongst other things, the main results derived here have experiment design implications for Hammerstein system estimation.
\end{abstract}

\section{Technical Report EE9933, Department of Electrical and Computer Engineering, University of Newcastle, AUSTRALIA}

\section{Introduction}

Over the last decade there has been intense activity devoted to quantifying the estimation error that arises due to undermodelling; see, for example, $[9,15,1,17,28,24]$ and the references therein.

However, as exposed by Guo and Ljung [5], when the undermodelling is due only to linear timeinvariant (LTI) model mismatch and is significant, then this indicates that model validation has not been adequately performed. Conversely, if undermodelling exists in the presence of proper model validation, then the estimation error involved must be no larger than any measurement-noise induced error, so that the total error can be measured by merely doubling the variance error quantification [5].

In recognition of this dispatch of the error quantification issue in the linear system case, the attention here is on non-linear systems; in particular, those of the Hammerstein class [25]. In this instance, if the non-linearity is ignored and only a linear model is fitted to the data, then the estimation bias is likely to be so large that any error bounds based on variance error quantifications will be strongly misleading.

For example, consider the case of fitting an $n=25^{\text {th }}$ order finite impulse response (FIR) model to input-output measurements of the system (sampling interval $T=1 \mathrm{~s}$ )

$$
G(q)=Z O H\left\{\frac{1}{(s+1)(2 s+1)}\right\}=\frac{0.1548 q+0.0939}{(q-0.6065)(q-0.3679)}
$$

when the input sequence $\left\{u_{t}\right\}$ is unit variance Gaussian distributed white noise and the output $\left\{y_{t}\right\}$ is corrupted by Gaussian white noise, with variance $\sigma^{2}=0.001$. Then the true and estimated linear systems (together with confidence bounds) for a typical data realisation are shown in figure 1(a). Clearly, since the true and estimated systems lie within the confidence regions, then these bounds (for

\footnotetext{
*This work was supported by the Australian Research Council and the Centre for Integrated Dynamics and Control. This author is with the Department of Electrical and Computer Engineering, University of Newcastle, Australia and can be contacted at email:brett@ee. newcast le.edu. au or FAX: +61 249216993

${ }^{\dagger}$ This author is also with the Department of Electrical and Computer Engineering, University of Newcastle, Australia and can be contacted at email:shgibson@ee. newcastle. edu. au or FAX: +61 249216993
} 


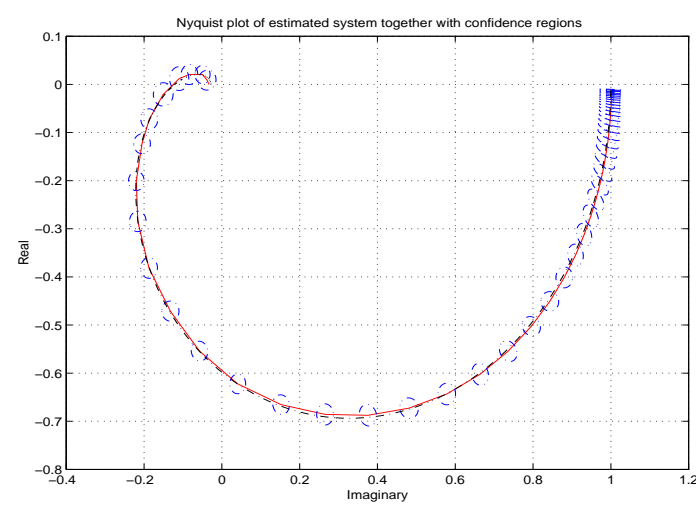

(a) FIR model fitted to linear system

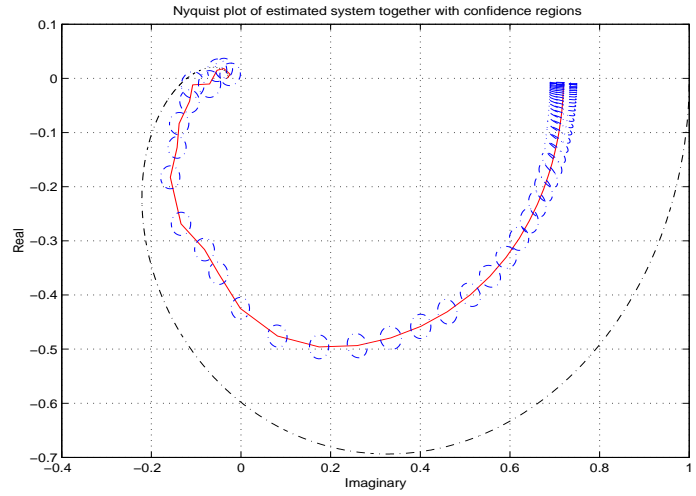

(b) FIR model fitted to Hammerstein system, confidence ellipses generated using noise variance

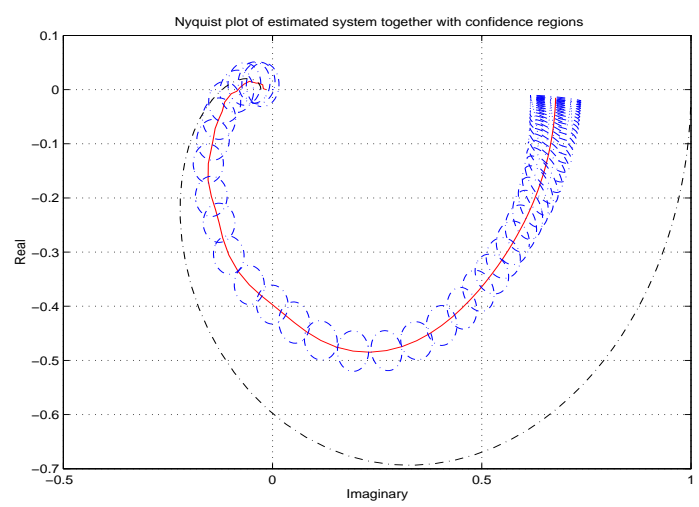

(c) Same as figure 1(b), but with confidence ellipses generated using estimated noise variance

Figure 1: Nyquist Plots of the true system (dot-dashed line) and the estimated FIR model (solid line). The ellipses are the 95\% confidence bounds associated with the FIR model. The top two (a),(b) plots involve using the true measurement noise variance in the computation of confidence ellipses, the bottom (c) plot uses an estimate of this variance formed from the prediction error residuals.

this data realisation) are an informative indication of overall model accuracy.

However, now suppose that the input is saturated symmetrically when its magnitude exceeds $\alpha=$ 1.0 so that the true system is of Hammerstein type [25]. In this case, if the same $25^{\text {th }}$ order linear FIR model is fitted to the data then (again for a typical realisation) the estimation results and confidence bounds are shown in figure 1(b). As can be seen, there is a dramatic difference between figures 1(a) and 1(b) in the reliability of the confidence region error quantifications. The error bound validity has been destroyed in figure 1(b) by estimation bias, which in turn has been induced by the model structure being linear and the true system being non-linear. The ellipsoids shown in 1(a) and 1(b) are true confidence regions in that the true measurement noise variance $\sigma^{2}=0.001$ has been used in computing them. However, it is also of interest to consider the more practical case in which this variance is estimated as a sample average from the prediction error residuals. This situation is shown in figure 1(c). Note that even though the error quantification has been enlarged by virtue of the prediction residuals accounting for undermodelling error as well as measurement noise, they still do 
not form an accurate quantification for the true estimation error.

In reaction to these sorts of difficulties, many investigations $[9,15,1,17,28,24]$ have sought to derive alternative error quantification methods that account for both the bias and variance error so that the error bounds in figure 1(b) may be replaced by more informative ones.

This paper is motivated by a different strategy of actually estimating the non-linearity (as well as the linear component) via a prediction error framework and then, since in this instance the true system is in the model class, again using confidence region error bounds as model uncertainty quantification (as in figure 1(a)).

A key concern in pursuing this approach involves the question: what price is paid, in terms of the quality of the linear system estimate, by including a parameterisation of a non-linear component in the model structure? Certainly any bias error should be eliminated via the model class being enriched to encompass the true system, but what of the variance error component which is included in the total error?

Put another way, while in recognition of the fact that a model is useless without accompanying (reliable) error bounds, the central goal of any system identification exercise must be to obtain the most accurate model possible. Is this achieved by ignoring non-linearities and then quantifying associated undermodelling errors, or is it achieved by including non-linearity in the model structure and then using a variance error quantification to evaluate the total error? Answering this latter question involves measuring how the variance error is affected by any non-linearities, and providing this quantification is the goal of the paper.

To further précis the work following, it is founded on a widely used approximate expression for the variability of an FIR model estimate $\widehat{G}(q)$ which was derived in [14] as

$$
\operatorname{Var}\left\{\widehat{G}\left(e^{j \omega}\right)\right\} \approx \frac{n}{N} \frac{\sigma^{2}}{\Phi_{u}(\omega)}
$$

Here $N$ is the length of the data records $\left\{y_{t}\right\}$ and $\left\{u_{t}\right\}, \Phi_{u}(\omega)$ is the spectral density of $\left\{u_{t}\right\}, \sigma^{2}$ is the variance of any white measurement noise, and $n$ is the order of the FIR model.

Furthermore, in the Hammerstein system case, the actual system input is a signal $x_{t}=X\left(u_{t}, \alpha\right)$ (where $X\left(u_{t}, \alpha\right)$ is a memoryless and possibly non-linear function of $u_{t}$ that is parameterised by a vector $\alpha$ ) so it would be natural to suppose that the effect of $X(\cdot, \alpha)$ would be to modify the variance error for the linear system estimate as

$$
\operatorname{Var}\left\{\widehat{G}\left(e^{j \omega}\right)\right\} \approx \frac{n}{N} \frac{\sigma^{2}}{\Phi_{x}(\omega)}
$$

where (of course) $\Phi_{x}(\omega)$ is the spectral density of $\left\{x_{t}\right\}$.

The main result of this paper is to show, both empirically and analytically, that in fact

$$
\operatorname{Var}\left\{\widehat{G}\left(e^{j \omega}\right)\right\} \approx \frac{1}{N}\left[\frac{n \sigma^{2}}{\Phi_{x}(\omega)}+\frac{1}{4}\left(\frac{\mathrm{d}}{\mathrm{d} \alpha} \log \Phi_{x}(\omega)\right)^{T} P_{\alpha}\left(\frac{\mathrm{d}}{\mathrm{d} \alpha} \log \Phi_{x}(\omega)\right)\left|\widehat{G}\left(e^{j \omega}\right)\right|^{2}\right]
$$

is a more accurate estimate of the variability of the linear FIR system estimate. Here $P_{\alpha} / N$ is the covariance matrix for the estimate of the parameterisation $\alpha$ of the nonlinearity, and the vector $\operatorname{dlog} \Phi_{x}(\omega) / \mathrm{d} \alpha$ measures the sensitivity of the spectrum of $X\left(u_{t}, \alpha\right)$ to changes in $\alpha$.

The key point is that (4) quantitatively establishes not only that identifying a non-linearity entails an unavoidable cost on the accuracy of the linear system estimate, but also that this cost actually depends on the dynamics of the linear part; compare this latter point with the expression (2) where the variability of $\widehat{G}$ is invariant to the true system $G$ itself. 


\section{Problem formulation}

The Hammerstein class of non-linear systems studied here may be represented by a model structure of the form $[16,23,25]$,

$$
y_{t}=G(q, \beta) x_{t}(\alpha)+e_{t}, \quad x_{t}(\alpha)=X\left(u_{t}, \alpha\right) .
$$

Here $X(\cdot, \alpha)$ is a memoryless non-linear function parameterised by the vector $\alpha$ and operating on the wide-sense stationry input $\left\{u_{t}\right\}$, while $\left\{e_{t}\right\}$ is an i.i.d., zero mean white noise process that represents corruptions in the output measurement $\left\{y_{t}\right\}$, and $G(q, \beta)$ is a linear and time invariant strictly stable system parameterised in the forward shift operator $q$ and by a vector $\beta$. The identification problem then becomes one of estimating the composite parameter vector

$$
\theta \triangleq\left[\beta^{T}, \alpha^{T}\right]^{T} ; \quad n \triangleq \operatorname{dim}\{\beta\}, \ell \triangleq \operatorname{dim}\{\alpha\}, m=n+\ell \triangleq \operatorname{dim}\{\theta\}
$$

on the basis of observations of $\left\{y_{t}\right\}$ and $\left\{u_{t}\right\}$. When it is necessary to be explicit about the dimensions of the parameter vectors, the symbols $\theta^{m}, \beta^{n}$ and $\alpha^{\ell}$ will be substituted for those given in (6); otherwise, in the interests of readability, these superscripts will be omitted.

For the solution of this estimation problem, a prediction-error or maximum likelihood approach $[10,21]$ may be taken in which the mean square optimal one step ahead predictor $\widehat{y}_{t}(\theta)$ is calculated as

$$
\widehat{y}_{t}(\theta)=G(q, \beta) x_{t}(\alpha)
$$

with the associated prediction error

$$
\varepsilon_{t}(\theta) \triangleq y_{t}-\widehat{y}_{t}(\theta)=y_{t}-G(q, \beta) x_{t}(\alpha) .
$$

In turn, this can be employed to define a "least-squares" estimation criterion of

$$
V_{N}(\theta)=\frac{1}{2 N} \sum_{t=1}^{N} \varepsilon_{t}^{2}(\theta)
$$

with an associated prediction error estimate (the $N$ subscript indicating dependence on $N$ data samples)

$$
\widehat{\theta}_{N} \triangleq \underset{\theta \in \mathbf{R}^{m}}{\arg \min } V_{N}(\theta)
$$

As an aside, when $\left\{e_{t}\right\}$ is Gaussian distributed then (modulo initial conditions) (10) is also the Maximum Likelihood estimate. However, under the much more relaxed assumptions of $\mathbf{E}\left\{e_{t}\right\}=0$, $\mathbf{E}\left\{e_{t}^{2}\right\}=\sigma^{2}<\infty$ and $\mathbf{E}\left\{\left|e_{t}\right|^{4+\delta}\right\}<\infty$ for some $\delta>0(\mathbf{E}\{\cdot\}$ denotes expectation over the probability space that any random variable components are defined upon), then the following strong consistency result is available $[10,11]$ (see also [8] for early work in this area)

$$
\lim _{N \rightarrow \infty} \widehat{\theta}_{N}=\theta_{\circ} \text { w.p.1, } \quad \theta_{\circ} \triangleq \underset{\theta \in \mathbf{R}^{m}}{\arg \min } \lim _{N \rightarrow \infty} \mathbf{E}\left\{V_{N}(\theta)\right\} .
$$

Furthermore, as precursed in the introduction, the error $G\left(e^{j \omega}, \widehat{\beta}_{N}\right)-G\left(e^{j \omega}\right)$ in the resulting estimate $G\left(e^{j \omega}, \widehat{\beta}_{N}\right)$ of any true underlying linear dynamics $G\left(e^{j \omega}\right)$ may be decomposed as $\left(\beta_{\circ}\right.$ is the component of $\theta_{\circ}$ defined in (11) consistent with (6))

$$
G\left(e^{j \omega}, \widehat{\beta}_{N}\right)-G\left(e^{j \omega}\right)=\left[G\left(e^{j \omega}, \widehat{\beta}_{N}\right)-G\left(e^{j \omega}, \beta_{\circ}\right)\right]+\left[G\left(e^{j \omega}, \beta_{\circ}\right)-G\left(e^{j \omega}\right)\right]
$$


where the last term (the so-called "bias error") is due to the estimation model structure (5) not being rich enough to encompass the true data generation mechanism, and the first term (the so-called "variance error") is due to the measurement noise.

As already mentioned, the quantification of the second term $G\left(e^{j \omega}, \beta_{\circ}\right)-G\left(e^{j \omega}\right)$ has already been the subject of much research during the last decade $[9,15,17,28]$. However, because of the challenging nature of the problem, it still appears to be open and hence the topic of ongoing investigation.

In recognition of this, the present work examines the following question: in situations where it is possible to extend the model structure richness so as to render the bias error $G\left(e^{j \omega}, \beta_{\circ}\right)-G\left(e^{j \omega}\right)$ negligible, then what price is paid in terms of overall error increase by virtue of a possible increase in the variance error $G\left(e^{j \omega}, \widehat{\beta}_{N}\right)-G\left(e^{j \omega}, \beta_{\circ}\right)$ ? For example, to continue the simulation example

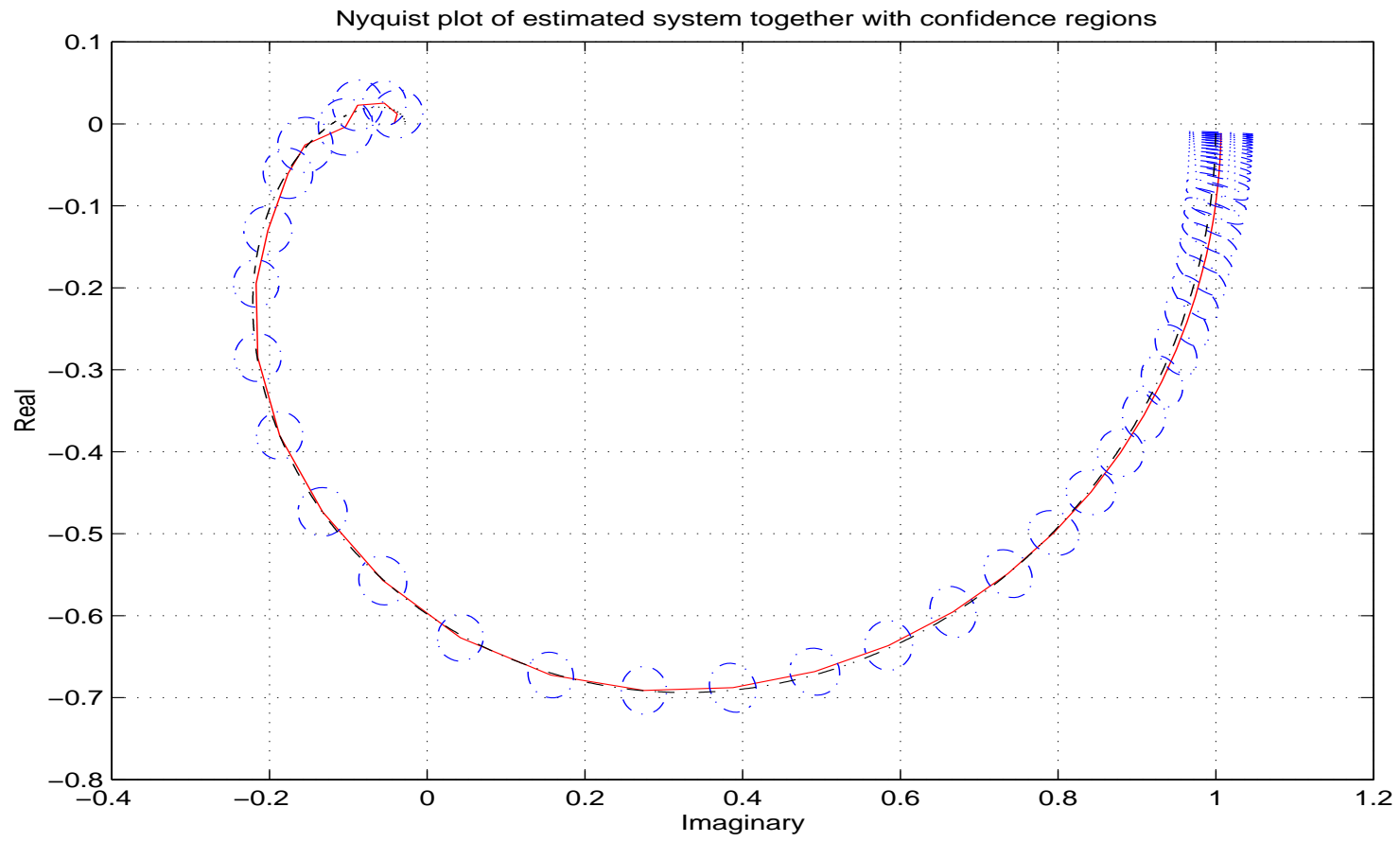

Figure 2: Nyquist plot of the (dash-dot line) true linear component within a Hammerstein structure together with its (solid line) least squares estimate when the latter forms part of a Hammerstein model structure. The ellipses are $95 \%$ confidence regions.

presented in the introduction, the bias error induced by ignoring a static input non-linearity is shown in figure 1(b) to be very large, but by way of contrast, if the possibility of this non-linearity is included in the model structure so that it may be estimated (via (5)-(10)), then the results are shown in figure 2.

Clearly, with the removal of the bias error, the overall error is now much smaller. But a price of increased variance error $G\left(e^{j \omega}, \widehat{\beta}_{N}\right)-G\left(e^{j \omega}, \beta_{\circ}\right)$ has been paid. That is, the $95 \%$ confidence ellipses that quantify the variance error are larger in figure 2 than in figure 1(b) where no non-linearity is present in the model structure.

The remainder of this paper is devoted to deriving (and then examining the consequences of) a simple approximate expression of engineering relevance that quantifies this variance error, and which has already been presented in equation (4). 


\section{Frequency Domain Analysis}

To analyse the question just introduced, the error quantification approach taken in this paper involves deriving frequency domain expressions, and this strategy has been inspired by its successful history [ 6 , $14,12]$.

Now, to this point, the discussion of the structure of the linear dynamics $G\left(q, \beta_{N}\right)$ has been focused on FIR models. In what follows, this paper will in fact address a generalisation expressible as

$$
G\left(q, \beta_{N}\right)=\sum_{k=1}^{n} \beta_{k} \mathcal{B}_{k}(q)
$$

where

$$
\mathcal{B}_{k}(q) \triangleq \frac{\sqrt{1-\left|\xi_{k}\right|^{2}}}{\left(q-\xi_{k}\right)} \prod_{r=1}^{k-1}\left(\frac{1-\overline{\xi_{r}} q}{q-\xi_{r}}\right), \quad \mathcal{B}_{1}(q) \triangleq \frac{\sqrt{1-\left|\xi_{1}\right|^{2}}}{q-\xi_{1}}
$$

The terms $\xi_{k} \in \mathbf{D} \triangleq\{|z| \in \mathbf{C}:|z|<1\}$ are user-chosen fixed poles in the linear model structure, and the selection $\xi_{k}=0, k=1,2, \ldots, n$ renders (12) as an FIR model as a special case. The motivation for allowing the flexibility of arbitrary fixed poles $\xi_{1}, \cdots, \xi_{n}$ is to allow the incorporation of prior knowledge into the estimation problem in the interests of increasing estimation accuracy $[7,26,18]$.

The use of such a model structure has a rich history, of which [7, 20, 26, 27] represents a recent part. In particular, and as discussed in [18], the expression (12) is precisely equivalent to a 'fixed denominator' model structure

$$
G^{\prime}\left(q, \beta_{N}^{\prime}\right)=\frac{\beta_{n}^{\prime} q^{n-1}+\cdots+\beta_{2}^{\prime} q+\beta_{1}^{\prime}}{\left(q-\xi_{1}\right)\left(q-\xi_{2}\right) \cdots\left(q-\xi_{n}\right)}
$$

in the sense that for any $\beta_{N}$ there exists a $\beta_{N}^{\prime}$ such that $G^{\prime}\left(q, \beta_{N}^{\prime}\right)=G\left(q, \beta_{N}\right)$. Furthermore, under the least-squares estimation criterion proposed (9) here, then (modulo numerical considerations) $G^{\prime}\left(q, \widehat{\beta}_{N}^{\prime}\right)=G\left(q, \widehat{\beta}_{N}\right)$.

The key motivation for considering the formulation (12) rather than the simpler one (14) is that the $\mathcal{B}_{k}(q)$ transfer functions in (12) are orthonormal in the sense that

$$
\left\langle\mathcal{B}_{k}, \mathcal{B}_{\ell}\right\rangle=\frac{1}{2 \pi} \int_{-\pi}^{\pi} \mathcal{B}_{k}\left(e^{j \omega}\right) \overline{\mathcal{B}_{\ell}\left(e^{j \omega}\right)} \mathrm{d} \omega=\delta(k-l)
$$

where $\delta$ is Kronecker's delta function. This property turns out to be essential in the development of certain results that have been derived in $[18,19]$ and will be employed here. That is, the orthonormal formulation (12) can be considered as an analysis tool that provides results pertinent to other (perhaps more direct) model structures such as (14).

With these comments on the model structure in mind, the following theorem provides a formal statement (together with formal proof) of the paper's main result.

Theorem 1. Consider the model structure defined by (5), (12) together with the prediction error estimate $G\left(q, \widehat{\beta}_{N}\right)$ defined by (5)-(10). Suppose that

1. The innovations $\left\{e_{t}\right\}$ satisfy $\boldsymbol{E}\left\{e_{t}\right\}=0, \boldsymbol{E}\left\{e_{t}^{2}\right\} \triangleq \sigma^{2}<\infty, \boldsymbol{E}\left\{\left|e_{t}\right|^{4+\delta}\right\}<\infty$ for some $\delta>0$, and $\left\{e_{t}\right\}$ is uncorrelated with the input $\left\{u_{t}\right\}$;

2. The signal $\left\{x_{t}\right\}$ defined in (5) together with $\left\{x_{t}^{\prime}\right\}$ defined as $x_{t}^{\prime} \triangleq \mathrm{d} X\left(u_{t}, \alpha\right) / \mathrm{d} \alpha$ are (jointly) wide-sense stationary with auto spectra $\Phi_{x}(\omega), \Phi_{x^{\prime}}(\omega)$ and cross spectrum $\Phi_{x x^{\prime}}(\omega)$ which are all Lipschitz continuous of some order $\eta>0$; 
3. The true system $G(q), X(u)$ is in the model class;

4. The available data is such that the model structure (5) is parameter identifiable.

Then remembering that $n=\operatorname{dim}\{\beta\}$ where $\beta$ parameterises the linear system estimate

$$
\begin{aligned}
& \lim _{n \rightarrow \infty} \lim _{N \rightarrow \infty} \frac{N}{K_{n}(\omega)} \boldsymbol{E}\left\{\left|G\left(e^{j \omega}, \widehat{\beta}_{N}^{n}\right)-G\left(e^{j \omega}, \beta_{\circ}^{n}\right)\right|^{2}\right\} \\
= & \frac{\sigma^{2}}{\Phi_{x}(\omega)}+\frac{1}{4}\left(\frac{\mathrm{d}}{\mathrm{d} \alpha} \log \Phi_{x}(\omega)\right)^{T} P_{\alpha}\left(\frac{\mathrm{d}}{\mathrm{d} \alpha} \log \Phi_{x}(\omega)\right) \times \lim _{n \rightarrow \infty} \frac{\left|G\left(e^{j \omega}, \beta_{\circ}^{n}\right)\right|^{2}}{K_{n}(\omega)}
\end{aligned}
$$

where

$$
\begin{aligned}
P_{\alpha} & \triangleq \lim _{N, n \rightarrow \infty} N \operatorname{Cov}\left\{\widehat{\alpha}_{N}\right\} \\
& =\sigma^{2}\left[\frac{1}{2 \pi} \int_{-\pi}^{\pi}\left|G\left(e^{j \omega}\right)\right|^{2}\left(\frac{\Phi_{x}(\omega) \Phi_{x^{\prime}}(\omega)-\Phi_{x x^{\prime}}^{\star}(\omega) \Phi_{x x^{\prime}}(\omega)}{\Phi_{x}(\omega)}\right) \mathrm{d} \omega\right]^{-1} \\
K_{n}(\omega) & \triangleq \sum_{k=1}^{n}\left|\mathcal{B}_{k}\left(e^{j \omega}\right)\right|^{2}=\sum_{k=1}^{n} \frac{1-\left|\xi_{k}\right|^{2}}{\left|e^{j \omega}-\xi_{k}\right|^{2}} .
\end{aligned}
$$

Proof. See Appendix A. The proof presented there rests on results developed in [19] for scalar functions, but which are extended in Appendix B to the case of vector valued functions. This is required in order to accommodate vector valued terms such as $\Phi_{x x^{\prime}}(\omega)$ when $\ell=\operatorname{dim}\{\alpha\}>1$.

\section{Ramifications}

There are several conclusions that can be drawn from Theorem 1 which are presented in the following subsections.

\subsection{Approximation of Estimation Errors}

The most direct result to be drawn from Theorem 1 is that, under an assumption that both $N$ and $n$ are large enough for the convergence in (15) to have essentially occurred, then multiplying both sides of (15) by $K_{n}(\omega) / N$ provides an approximation of the variance error as

$\operatorname{Var}\left\{G\left(e^{j \omega}, \widehat{\beta}_{N}\right)\right\} \approx \frac{1}{N}\left[\frac{\sigma^{2} K_{n}(\omega)}{\Phi_{x}(\omega)}+\frac{1}{4}\left(\frac{\mathrm{d}}{\mathrm{d} \alpha} \log \Phi_{x}(\omega)\right)^{T} P_{\alpha}\left(\frac{\mathrm{d}}{\mathrm{d} \alpha} \log \Phi_{x}(\omega)\right)\left|G\left(e^{j \omega}, \widehat{\beta}_{N}\right)\right|^{2}\right]$.

Here $P_{\alpha}$ is the covariance matrix for the (scaled) estimate $\sqrt{N} \widehat{\alpha}_{N}$ of the parameterisation of the memory-less input non-linearity, which from Theorem 1 may also be approximated by the frequency domain expression

$$
\operatorname{Var}\left\{\widehat{\alpha}_{N}\right\}=\frac{P_{\alpha}}{N} \approx \frac{\sigma^{2}}{N}\left[\frac{1}{2 \pi} \int_{-\pi}^{\pi}\left|G\left(e^{j \omega}, \widehat{\beta}_{N}\right)\right|^{2}\left(\frac{\Phi_{x}(\omega) \Phi_{x^{\prime}}(\omega)-\Phi_{x x^{\prime}}^{\star}(\omega) \Phi_{x x^{\prime}}(\omega)}{\Phi_{x}(\omega)}\right) \mathrm{d} \omega\right]^{-1} .
$$

Of course, it is obvious to question the accuracy of such approximating procedures. In particular, how large should $N$ and $n$ be for (19) and (20) to be an informative and accurate quantification?

Answering this question with full rigor would involve providing hard error bounds on the differences between the left and right hand sides of (19) and (20), and this would appear intractable. As 
an alternative, this paper adopts an approach in $\S 6$ (also taken in related works $[12,14,18]$ ) of examining the issue via Monte-Carlo simulation examples. The ensuing results provide evidence that, in fact, (19) and (20) can provide a highly accurate approximation in cases of quite moderate data length $N$ and model order $n$ that are consistent with what would typically be encountered in engineering practice.

Furthermore, as presented in detail in [18], a key reason for employing the orthonormal parameterisation (12) (as opposed to a more direct one such as (14)) is to accelerate the convergence (in $n$ ) of (19) by means of choosing a basis that is 'adapted' to the quantities that are to be approximated. A manifestation of this approach is the presence of the weighting term $K_{n}(\omega)$ in (19) which is determined solely by the choice of fixed poles $\xi_{1}, \cdots, \xi_{n}$ in the model structure. In the special FIR case of $\xi_{k}=0$, then $K_{n}(\omega)=n$ in which case (19) becomes the expression (4) used in the introduction.

\subsection{Identifiability of the memoryless non-linearity}

Another interesting aspect of Theorem 1 is that it provides insight into when the non-linearity parameterised by $\alpha$ is identifiable. Specifically, the spectral weighting in the definition (17) for $P_{\alpha}$ shows that identifiability is lost $\left(P_{\alpha}\right.$ becomes unbounded) whenever $\Phi_{x}(\omega) \Phi_{x^{\prime}}(\omega)=\Phi_{x x^{\prime}}^{\star}(\omega) \Phi_{x x^{\prime}}(\omega)$ for all $\omega$ and locally around $\alpha_{\circ}$ (which is defined via $\theta_{\circ}$ in (11)).

To gain understanding of this principle, notice that if there is a linear relationship $x_{t}^{\prime}\left(\alpha_{\circ}\right)=$ $K(q) x_{t}\left(\alpha_{\circ}\right)$ for some stable $K(q)$, then for the case in which $\operatorname{dim}\{\alpha\}=\ell=1$,

$$
\left|\Phi_{x^{\prime} x}\right|^{2}=\left|K\left(e^{j \omega}\right)\right|^{2} \Phi_{x}^{2}(\omega)=\left(\left|K\left(e^{j \omega}\right)\right|^{2} \Phi_{x}(\omega)\right) \Phi_{x}(\omega)=\Phi_{x^{\prime}}(\omega) \Phi_{x}(\omega)
$$

so that $\Phi_{x}(\omega) \Phi_{x^{\prime}}(\omega)-\Phi_{x x^{\prime}}^{\star}(\omega) \Phi_{x x^{\prime}}(\omega)=0$. Therefore, the weighting

$$
\frac{\Phi_{x}(\omega) \Phi_{x^{\prime}}(\omega)-\left|\Phi_{x^{\prime} x}(\omega)\right|^{2}}{\Phi_{x}(\omega)}
$$

can be interpreted as a measure of the non-linearity of the relationship between $x_{t}(\alpha)$ and $x_{t}^{\prime}(\alpha)$.

That is, the approximate expression (20) indicates that the accuracy of the estimate of the nonlinear component increases (its variance decreases) as the mapping between $x_{t}=X\left(u_{t}, \alpha\right)$ and its sensitivity $x_{t}^{\prime}=\mathrm{d} X\left(u_{t}, \alpha\right) / \mathrm{d} \alpha$ to changes in $\alpha$ becomes 'more non-linear', and in the case in which the mapping is purely linear, then the accuracy becomes so poor that in fact identifiability is lost.

This general principle provides, for example, a direct means to derive (the well known fact) that PRBS signals cannot be used for the identification of dead-zones or saturations; see $\S 5.1$ following.

It is important to clarify that this discussion of identifiability has considered the memoryless nonlinearity in isolation. A related, but different issue is that of the identifiability of the overall parameterisation $\theta^{T}=\left[\beta^{T}, \alpha^{T}\right]$ for the combined linear and non-linear model structure components.

For example, the model $X\left(u_{t}, \alpha\right)$ might (in isolation) be parameter identifiable, but if it is such that for any $\zeta \in \mathbf{R}$ there always exists two parameters $\alpha_{1}, \alpha^{2}$ such that

$$
X\left(\zeta u_{t}, \alpha_{1}\right)=X\left(u_{t}, \alpha_{2}\right)
$$

then estimation of an overall gain term is involved. This implies that if used in combination with a subsequent linear structure $G(q, \beta)$ that also allows for estimation of an overall gain, then the joint parameterisation $\theta^{T}=\left[\beta^{T}, \alpha^{T}\right]$ cannot hope to be identifiable. The results of this paper do not address this joint identifiability issue, and indeed condition 4 of Theorem 1 assumes joint identifiability. 


\subsection{Interaction between Linear and Non-linear components}

The approximations (19) and (20) reveal how the estimation accuracies of the linear and non-linear components of a Hammerstein model structure are inter-twined, and in turn this provides insight into some unique aspects of the estimation problem for Hammerstein model structures.

Firstly, it should be pointed out that a key conclusion to arise from the seminal work $[12,14]$ is the fundamental principle that the variability of a linear system estimate is invariant to the true linear dynamics, and instead (see (2)) depends only on the experimental conditions of data length $N$, chosen model order $n$ and signal-to-noise ratio [10].

In contrast, the expression (19), by way of the term

$$
\left(\frac{\mathrm{d}}{\mathrm{d} \alpha} \log \Phi_{x}(\omega)\right)^{T} P_{\alpha}\left(\frac{\mathrm{d}}{\mathrm{d} \alpha} \log \Phi_{x}(\omega)\right)\left|G\left(e^{j \omega}, \widehat{\beta}_{N}\right)\right|^{2}
$$

shows that in the Hammerstein model case, the estimation accuracy is far from system-invariant. Instead (because of the component $\left|G\left(e^{j \omega}, \widehat{\beta}_{N}\right)\right|^{2}$ ), it depends on the true linear dynamics as well as (because of the component $\operatorname{dlog} \Phi_{x}(\omega) / \mathrm{d} \alpha$ ) the static input non-linearity, and an explanation for why this phenomenon occurs is provided in $\S 4.4$ following.

Indeed, as will be illustrated in $\S 6$ following, the term (21) can easily dominate the complete expression (19). In particular, the presence of the log term means that (21) will be likely to play a significant role in (19) in frequency regions where $\Phi_{x}(\omega)$ is small.

Turning now to the expression (20) quantifying the accuracy of the non-linear block estimate, note that it too is far from invariant to the true underlying system. Firstly, by means of the $\left|G\left(e^{j \omega}, \widehat{\beta}_{N}\right)\right|^{2}$ term, it clearly depends on the linear dynamics. More specifically, (20) indicates that in order for the estimate $\widehat{\alpha}_{N}$ to be accurate, then $\mid G\left(\left.e^{j \omega}\right|^{2}\right.$ must have reasonable gain in frequency regions in which the mapping between $\Phi_{x}$ and $\Phi_{x^{\prime}}$ indicates non-linearity between $x_{t}$ and $x_{t}^{\prime}$.

Perhaps more suprisingly though, the expression (20) shows that the accuracy with which $\widehat{\alpha}_{N}$ may be estimated, is not dependent on the total number of parameters that are estimated. To be specific, in the purely linear system case, expressions such as (2) illustrate a principle that the estimation accuracy decreases as more parameters $n$ are estimated. In contrast, the quantification (20) is invariant to $n$, and thus there is a decoupling in that the accuracy of the estimate of the non-linear component is not affected by the complexity of the model structure used to estimate the linear component. This principle is illustrated via simulation example in figure 7(b).

\subsection{Measurement Error to Frequency Domain Error: A General Principle}

Clearly, as just discussed, the expression (19) resulting from Theorem 1 indicates several aspects of achievable estimation accuracy that are quite different to what might be expected from previous studies of linear system identification.

In turn, this naturally leads to the question 'is there a more general principle applying, that can encompass a wide variety of cases, and lead to results such as (2) and (19) as special cases?'.

This section hypothesises and then tests such a general principle, and by way of this provides an explanation of the underlying mechanism for some of the conclusions made in $\S 4.1-4.3$. The principle proposed here is that the previous results are hypothesised to be manifestations of a general principle:

The relative frequency domain estimation error times $N$ is equal to the relative sensitivity of the model-based prediction $\widehat{y}$ to the model parameters.

That is, by denoting relative estimation error as $\mathrm{d} \widehat{G} / \widehat{G}$ and relative sensitivity of model predictions as $\mathrm{d} \widehat{y} / \widehat{y}$, then one could hypothesise that the well known approximation (2) together with the new 
one (19) are both manifestations of a more fundamental principle which ensures that the following equation holds:

$$
N \frac{\operatorname{Var}\{\widehat{G}\}}{|\widehat{G}|^{2}} \equiv N \frac{|\mathrm{d} \widehat{G}|^{2}}{|\widehat{G}|^{2}}=\frac{(\mathrm{d} \widehat{y})^{2}}{\widehat{y}^{2}} .
$$

To test this hypothesis, it is now shown to lead to (19), (and hence also (2)) as a special case. Firstly, on noting that according to the model structure $\widehat{y}_{t}=G(q, \beta) x_{t}(\alpha)$ then (22) becomes

$$
N|\mathrm{~d} \widehat{G}|^{2}=\frac{(\mathrm{d} \widehat{y})^{2}}{\widehat{y}^{2}}|\widehat{G}|^{2}=\frac{(\mathrm{d} \widehat{y})^{2}}{|\widehat{G}|^{2} x^{2}}|\widehat{G}|^{2}=\frac{(\mathrm{d} \widehat{y})^{2}}{x^{2}} .
$$

Now, in the Hammerstein case, there are two factors contributing to $\mathrm{d} \widehat{y}$ - sensitivities of $G(q, \beta)$ to $\beta$ and sensitivities of $X(\cdot, \alpha)$ to $\alpha$ so that $(\mathrm{d} \widehat{y})^{2}=(\mathrm{d} \widehat{G})^{2} x^{2}+|\widehat{G}|^{2}(\mathrm{~d} x)^{2}$. Therefore, assuming for simplicity that $\alpha$ is scalar

$$
\begin{aligned}
N|\mathrm{~d} \widehat{G}|^{2}=\frac{(\mathrm{d} \widehat{y})^{2}}{x^{2}} & =\frac{1}{x^{2}}\left[(\mathrm{~d} \widehat{G})^{2} x^{2}+|\widehat{G}|^{2}(\mathrm{~d} x)^{2}\right] \\
& =\frac{1}{x^{2}}\left[\left\|\frac{\mathrm{d} \widehat{G}}{\mathrm{~d} \beta}\right\|^{2}\|\mathrm{~d} \beta\|^{2} x^{2}+|\widehat{G}|^{2}\left(\frac{\mathrm{d} x}{\mathrm{~d} \alpha}\right)^{2}(\mathrm{~d} \alpha)^{2}\right] .
\end{aligned}
$$

Now, according to the model structure (12), $\mathrm{d} G(q, \beta) / \mathrm{d} \beta_{k}=\mathcal{B}_{k}(q)$ so that $\left\|\mathrm{d} G\left(e^{j \omega}, \beta\right) / \mathrm{d} \beta\right\|^{2}=$ $\sum_{k}\left|\mathcal{B}_{k}\left(e^{j \omega}\right)\right|^{2}=K_{n}(\omega)$. Furthermore, with the measurement $\|\mathrm{d} \beta\|^{2} \triangleq \mathbf{E}\left\{\left\|\widehat{\beta}_{N}-\beta_{\circ}\right\|^{2}\right\}$ then via a well-known [10] Taylor series expansion argument $\left(\left(\cdot^{\prime}\right)\right.$ denotes differentiation with respect to $\beta$ about the point $\beta_{\circ}$ )

$$
\|\mathrm{d} \beta\|^{2}=\operatorname{Tr}\left\{\left[\mathbf{E}\left\{V_{N}^{\prime \prime}\right\}\right]^{-1}\left[\mathbf{E}\left\{V_{N}^{\prime}\right\}\right]\left[\mathbf{E}\left\{V_{N}^{\prime \prime}\right\}\right]^{-1}\right\}=\sigma^{2} \operatorname{Tr}\left\{\left[\mathbf{E}\left\{V_{N}^{\prime \prime}\right\}\right]^{-1}\right\}=\frac{\sigma^{2}}{\Phi_{x}}
$$

where the second equality follows by means of the assumption of the true system being in the model class, and the third equality follows from a well known spectral representation of $\left.\mathbf{E}\left\{V_{N}^{\prime \prime}\right\}\right][10,14]$.

Using these expressions in (24) together with the measurements $(\mathrm{d} \alpha)^{2}=\mathbf{E}\left\{\left(\widehat{\alpha}_{N}-\alpha_{\circ}\right)^{2}\right\}=P_{\alpha}$ and $x^{2}=\Phi_{x}$ then provides

$$
\begin{aligned}
N|\mathrm{~d} G|^{2} & =K_{n}(\omega) \frac{\sigma^{2}}{\Phi_{x}(\omega)}+|G|^{2} \frac{1}{4 x^{4}}\left(\frac{\mathrm{d} x^{2}}{\mathrm{~d} \alpha}\right)^{2}(\mathrm{~d} \alpha)^{2} \\
& =K_{n}(\omega) \frac{\sigma^{2}}{\Phi_{x}(\omega)}+|G|^{2} P_{\alpha}\left(\frac{1}{2} \frac{\mathrm{d}}{\mathrm{d} \alpha} \log \Phi_{x}(\omega)\right)^{2}
\end{aligned}
$$

which is the quantification (19).

Therefore, the presence of the (perhaps unexpected) term $\left|G\left(e^{j \omega}, \widehat{\beta}_{N}\right)\right|^{2}$ arises, according to the hypothesis (22), because uncertainties in the nature of the non-linearity $X(\cdot, \alpha)$ are mapped through $G\left(q, \widehat{\beta}_{N}\right)$ before manifesting themselves in the output prediction error.

\section{Analytical Examples}

Clearly, a key issue in using the results of Theorem 1 is that of understanding the nature of the spectral densities $\Phi_{x}(\omega), \Phi_{x^{\prime}}(\omega)$ and $\Phi_{x x^{\prime}}(\omega)$. Because these densities arise via signals passed through a nonlinearity $X(\cdot, \alpha)$, the question of evaluating them may be non-trivial. 
However, consider the case when $\left\{u_{t}\right\}$ is i.i.d. with $\Phi_{u}(\omega)$ then a constant. In this case, since it is well known that an arbitrary (measurable) function of an i.i.d. process yields an i.i.d. process (see Theorem B.9), then $\left\{x_{t}(\alpha)\right\},\left\{x_{t}^{\prime}(\alpha)\right\}$ are both i.i.d. and hence

$$
\Phi_{x}(\omega) \triangleq \sigma_{x}^{2}, \quad \Phi_{x^{\prime}}(\omega) \triangleq \sigma_{x^{\prime}}^{2} \quad \text { and } \quad \Phi_{x x^{\prime}}(\omega) \triangleq \sigma_{x x^{\prime}}^{2}
$$

are all constants.

The question then arises as to how these constants $\sigma_{x}^{2}, \sigma_{x^{\prime}}^{2}$ and $\sigma_{x x^{\prime}}^{2}$ all relate to the input spectrum $\Phi_{u}(\omega)$ and the nature of the non-linearity $X(\cdot, \alpha)$. A general discussion of this issue quickly becomes very complicated [22], so in an effort to maximise insight, this paper proceeds by considering the two specific (and common) non-linearities of saturation and dead-zone. As well, in the interests of concreteness, specific distributions for the i.i.d. input $\left\{u_{t}\right\}$ will be supposed.

\subsection{Saturation Non-linearity}

The saturation non-linearity may be specified (for $\alpha_{2}<\alpha_{1}$ ) as follows:

$$
X(u, \alpha)= \begin{cases}u & ; u \in\left[\alpha_{2}, \alpha_{1}\right] \\ \alpha_{1} & ; u<\alpha_{1} \\ \alpha_{2} & ; u>\alpha_{2}\end{cases}
$$

In this case, suppose that the input $\left\{u_{t}\right\}$ is i.i.d. with uniform density defined by:

$$
p\left(u_{t}\right)= \begin{cases}\frac{1}{2 \lambda} & ;\left|u_{t}\right| \leq \lambda \\ 0 & ; \text { otherwise. }\end{cases}
$$

Then for this scenario the spectral densities required in the approximation (15) are provided by the following Lemma.

Lemma 5.1. For the case of a saturation non-linearity (26) which is symmetric in that $\alpha \triangleq \alpha_{1}=-\alpha_{2}$ (see figure 3(a)) and for a uniformly distributed i.i.d. input $\left\{u_{t}\right\}$ according to (27), then for $\lambda>\alpha$ :

$$
\Phi_{x}(\omega)=\frac{\alpha^{2}}{3 \lambda}(3 \lambda-2 \alpha), \quad \Phi_{x x^{\prime}}(\omega)=\alpha\left(\frac{\lambda-\alpha}{\lambda}\right), \quad \Phi_{x^{\prime}}(\omega)=\frac{\lambda-\alpha}{\lambda} .
$$

Proof. Clearly, when $\lambda>\alpha$ (otherwise $\mathbf{E}\left\{x_{t}^{2}(\alpha)\right\}=\mathbf{E}\left\{u_{t}^{2}\right\}=\lambda^{2} / 3$ ),

$$
\begin{aligned}
\mathbf{E}\left\{x_{t}^{2}(\alpha)\right\} & =\int_{-\infty}^{\infty} X^{2}(u, \alpha) p(u) \mathrm{d} u \\
& =\frac{1}{2 \lambda}\left(\int_{-\alpha}^{\alpha} u^{2} \mathrm{~d} u+\int_{\alpha}^{\lambda} \alpha^{2} \mathrm{~d} u+\int_{\lambda}^{-\alpha} \alpha^{2} \mathrm{~d} u\right) \\
& =\frac{\alpha^{2}}{3 \lambda}(3 \lambda-2 \alpha) .
\end{aligned}
$$

Furthermore, on consideration of figure 3(b) which shows $\mathrm{d} X\left(u_{t}, \alpha\right) / \mathrm{d} \alpha$ plotted as a function of $u$, for $\lambda>\alpha$ (otherwise $\mathbf{E}\left\{x_{t}^{\prime}(\alpha)^{2}\right\}=0$ )

$$
\begin{aligned}
\mathbf{E}\left\{\left[x_{t}^{\prime}(\alpha)\right]^{2}\right\} & =\int_{-\infty}^{\infty}\left[\frac{\mathrm{d} X(u, \alpha)}{\mathrm{d} \alpha}\right]^{2} p(u) \mathrm{d} u \\
& =\int_{\alpha}^{\infty} p(u) \mathrm{d} u+\int_{-\infty}^{-\alpha} p(u) \mathrm{d} u \\
& =1-\frac{\alpha}{\lambda} .
\end{aligned}
$$


Finally, again on consideration of figure 3 , for $\lambda>0$ (otherwise $\mathbf{E}\left\{x_{t}(\alpha) x_{t}^{\prime}(\alpha)\right\}=0$ )

$$
\mathbf{E}\left\{x_{t}(\alpha) x_{t}^{\prime}(\alpha)\right\}=\int_{\alpha}^{\lambda} \alpha p(u) \mathrm{d} u+\int_{-\lambda}^{-\alpha} \alpha p(u) \mathrm{d} u=\alpha\left(1-\frac{\alpha}{\lambda}\right) .
$$

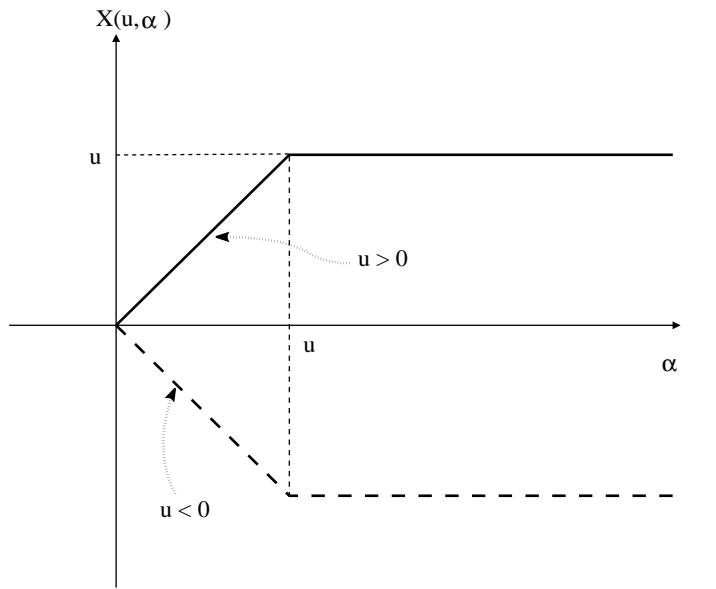

(a) Symmetric saturation output as a function of saturation level $(\alpha)$

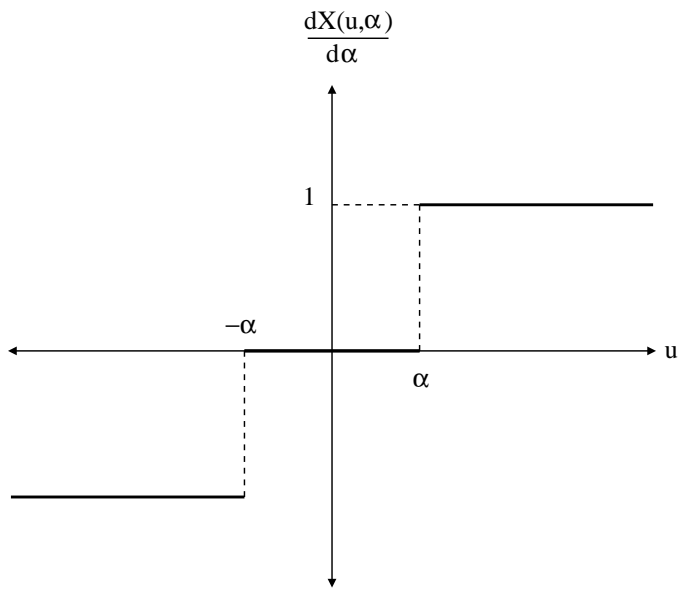

(b) Sensitivity of the output of a symmetric saturation function to its saturation level $(\alpha)$

Figure 3: Symmetric Saturation Function.

Using these expressions in (19) then gives the variance approximation

$$
\mathbf{E}\left\{\left|G\left(e^{j \omega}, \widehat{\beta}_{N}\right)-G\left(e^{j \omega}, \beta_{\circ}^{n}\right)\right|^{2}\right\} \approx \frac{3 \sigma^{2}}{\alpha^{2} N} \frac{\lambda}{(3 \lambda-2 \alpha)}\left[K_{n}(\omega)+\frac{3\left|G\left(e^{j \omega}, \beta_{\circ}\right)\right|^{2}}{\|G\|^{2}}\left(\frac{\lambda-\alpha}{\alpha}\right)\right],
$$

where

$$
\|G\|^{2} \triangleq \frac{1}{2 \pi} \int_{-\pi}^{\pi}\left|G\left(e^{j \omega}, \beta_{\circ}\right)\right|^{2} \mathrm{~d} \omega
$$

An important conclusion that can be drawn from (28) (apart from the fact that it depends on $\left|G\left(e^{j \omega}, \beta_{\circ}\right)\right|$, which has already been commented on) is that it indicates the existence of an optimal input excitation power.

That is, in the linear case, the estimation accuracy is proportional to $1 / \Phi_{u}(\omega)$ so that the more input excitation power, the better. However, as shown by (28), in the non-linear case with input saturation, (neglecting the common constants) the overall variability consists of two terms, one of which

$$
\frac{K_{n}(\omega) \lambda}{(3 \lambda-2 \alpha)}
$$

decreases monotonically with increasing input power $\Phi_{u}(\omega)=\lambda^{2} / 3$, and the second term of which

$$
\frac{3 \lambda}{(3 \lambda-2 \alpha)}\left(\frac{\lambda-\alpha}{\alpha}\right) \frac{\left|G\left(e^{j \omega}, \beta_{\circ}\right)\right|^{2}}{\|G\|^{2}}
$$


increases monotonically with increasing $\Phi_{u}(\omega)=\lambda^{2} / 3$. As a result, for every frequency $\omega$ there is an optimal input power (in the sense of minimising the variability of the estimate) given by the solution of

$$
\left(3 t^{2}-4 t+2\right)=\frac{2}{3} \frac{\|G\|^{2}}{\left|G\left(e^{j \omega}, \beta_{\circ}\right)\right|^{2}} K_{n}(\omega)
$$

where $t=\lambda / \alpha$.

At first, this principle that an optimal input power exists, and hence that increasing the input power may lead to a degraded estimate, might seem counter-intuitive. However, once exposed via (15) and (28), it is undersood to arise from the fact that as $\lambda$ is increased, the probability of a realisation of $u_{t}$ being below the saturation level is decreased and hence an increased $\lambda$ leads to an input sequence $\left\{u_{t}\right\}$ which provides less 'exploration' of the non-linearity. As a result, the variability of the estimate is increased. Vice-versa, if $\lambda$ is too small (only slightly larger than $\alpha$ ), then again the non-linearity is not explored since there is a low probability that $u_{t}$ will explore the saturation limit $\alpha$.

Another common excitation used in the practice of system identification is the Pseudo-Random Binary Noise Sequence (PRBS) [10]. This can be modelled as an i.i.d. process with binomial (discrete) density

$$
p\left(u_{t}\right)= \begin{cases}0.5 & ; u_{t}=\lambda \\ 0.5 & ; u_{t}=-\lambda .\end{cases}
$$

In this case the required spectral densities are as follows.

Lemma 5.2. For $\left\{u_{t}\right\}$ i.i.d. with binomial distribution (30) and $X\left(u_{t}, \alpha\right)$ being the saturation nonlinearity (26) which is symmetric $\left(\alpha \triangleq \alpha_{1}=-\alpha_{2}\right)$ then for $\lambda>\alpha$

$$
\Phi_{x}(\omega)=\alpha^{2}, \quad \Phi_{x x^{\prime}}(\omega)=\alpha, \quad \Phi_{x^{\prime}}(\omega)=1 .
$$

Proof.

$$
\begin{aligned}
\mathbf{E}\left\{x_{t}(\alpha)^{2}\right\} & =0.5 \times \alpha^{2}+0.5 \times \alpha^{2}=\alpha^{2}, \\
\mathbf{E}\left\{x_{t}(\alpha) x_{t}^{\prime}(\alpha)\right\} & =1 \times \alpha \times 0.5+(-1) \times(-\alpha) \times 0.5=\alpha, \\
\mathbf{E}\left\{\left[x_{t}^{\prime}(\alpha)\right]^{2}\right\} & =1^{2} \times 0.5+(-1)^{2} \times 0.5=1
\end{aligned}
$$

Therefore, in the PRBS case, a difficulty is encountered in that the term $\Phi_{x}(\omega) \Phi_{x^{\prime}}(\omega)-\left|\Phi_{x x^{\prime}}(\omega)\right|^{2}$ arising in the component $P_{\alpha}$ of (15) becomes:

$$
\Phi_{x}(\omega) \Phi_{x^{\prime}}(\omega)-\left|\Phi_{x x^{\prime}}(\omega)\right|^{2}=\alpha^{2}-\alpha^{2}=0,
$$

and hence the second term in (15) is infinite.

This problem is a manifestation of the phenomenon introduced in $\S 3$, wherein it is noted that the complete linear/non-linear structure of a Hammerstein system is identifiable only if the mapping between $x_{t}(\alpha)$ and $x_{t}^{\prime}(\alpha)$ is non-linear.

That is, even though a PRBS input is widely and successfully used for estimating purely linear systems, it is inappropriate when saturations are involved since in this case the effect of the nonlinearity can be described as

$$
X\left(u_{t}, \alpha\right)=\frac{\alpha}{\lambda} u_{t} \quad ; \lambda>\alpha
$$

and hence the non-linearity actually appears to be a linear gain.

While, once made, this conclusion appears obvious (and is already well known), the fact that it is embedded in the variance approximation (15) highlights the utility of that expression. 


\subsection{Dead-zone Non-linearity}

This non-linearity has the following mathematical description (for $\alpha_{2}<\alpha_{1}$ ):

$$
X(u, \alpha)= \begin{cases}u-\alpha_{1} & ; u>\alpha_{1}, \\ 0 & ; u \in\left[\alpha_{2}, \alpha_{1}\right], \\ u+\alpha_{2} & ; u<\alpha_{2} .\end{cases}
$$

Repeating the sort of analysis conducted for the case of a saturation non-linearity now proceeds as follows:

Lemma 5.3. For the case of the dead-zone non-linearity (31) which is symmetric in that $\alpha \triangleq \alpha_{1}=$ $-\alpha_{2}$ (see figure $\left.4(a)\right)$ and independent, uniformly distributed input $\left\{u_{t}\right\}$ according to (27) then for $\lambda>\alpha$

$$
\Phi_{x}(\omega)=\frac{(\lambda-\alpha)^{3}}{3 \lambda}, \quad \Phi_{x^{\prime}}(\omega)=1-\frac{\alpha}{\lambda}, \quad \Phi_{x x^{\prime}}(\omega)=-\frac{(\lambda-\alpha)^{2}}{2 \lambda}
$$

Proof. When $\lambda>0$ (otherwise $\mathbf{E}\left\{x_{t}^{2}(\alpha)\right\}=0$ )

$$
\begin{aligned}
\mathbf{E}\left\{x_{t}^{2}(\alpha)\right\} & =\int_{-\infty}^{\infty} X^{2}(u, \alpha) p(u) \mathrm{d} u \\
& =\int_{\alpha}^{\lambda}(u-\alpha)^{2} p(u) \mathrm{d} u+\int_{-\lambda}^{-\alpha}(\alpha+u)^{2} p(u) \mathrm{d} u \\
& =\frac{(\lambda-\alpha)^{3}}{3 \lambda} .
\end{aligned}
$$

Furthermore, on consideration of figure 3, for $\lambda>\alpha$ (otherwise $\mathbf{E}\left\{\left[x_{t}^{\prime}(\alpha)\right]^{2}\right\}=0$ )

$$
\begin{gathered}
\mathbf{E}\left\{\left[x_{t}^{\prime}(\alpha)\right]^{2}\right\}=\int_{-\infty}^{\infty}\left[\frac{\mathrm{d} X(u, \alpha)}{\mathrm{d} \alpha}\right]^{2} p(u) \mathrm{d} u \\
=\int_{\alpha}^{\infty} p(u) \mathrm{d} u+\int_{-\infty}^{-\alpha} p(u) \mathrm{d} u \\
=\frac{1}{2 \lambda} \int_{\alpha}^{\lambda} \mathrm{d} u+\frac{1}{2 \lambda} \int_{-\lambda}^{-\alpha} \mathrm{d} u=1-\frac{\alpha}{\lambda} . \\
\mathbf{E}\left\{x_{t}(\alpha) x_{t}^{\prime}(\alpha)\right\}=\int_{\alpha}^{\infty}(u-\alpha)(-1) p(u) \mathrm{d} u+\int_{-\infty}^{-\alpha}(u+\alpha)(+1) p(u) \mathrm{d} u \\
=\frac{-1}{2 \lambda}\left(\int_{\alpha}^{\lambda}(u-\alpha) \mathrm{d} u-\int_{-\lambda}^{-\alpha}(u+\alpha) \mathrm{d} u\right) \\
=\frac{-(\lambda-\alpha)^{2}}{2 \lambda} .
\end{gathered}
$$

Therefore,

$$
\frac{\Phi_{x}(\omega)}{\Phi_{x}(\omega) \Phi_{x^{\prime}}(\omega)-\left|\Phi_{x^{\prime} x}(\omega)\right|^{2}}=\frac{4 \lambda}{\lambda-\alpha}
$$




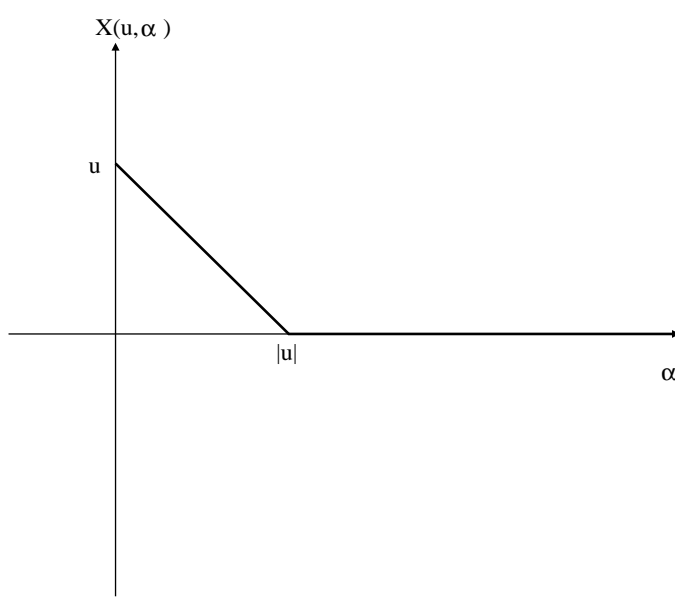

(a) Symmetric dead-zone output as a function of dead-zone level $(\alpha)$

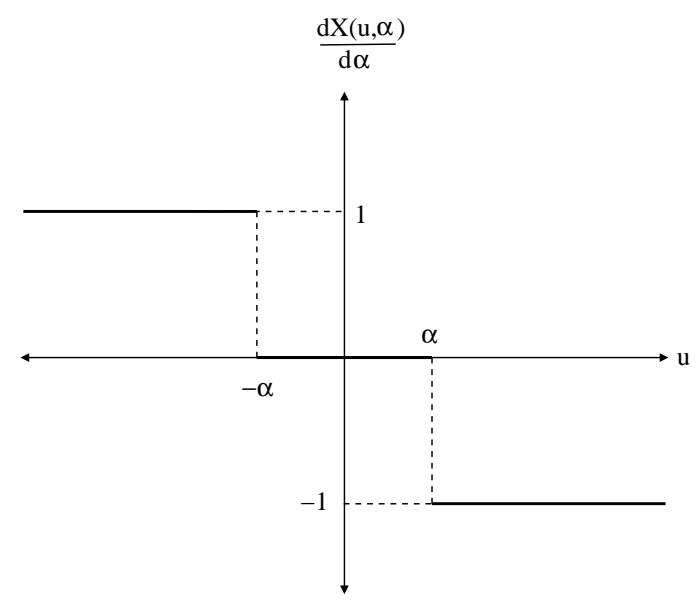

(b) Sensitivity of the output of a symmetric deadzone function to its deadzone level $(\alpha)$

Figure 4: Symmetric Dead-zone Function.

$$
\left(\frac{1}{2} \frac{\mathrm{d}}{\mathrm{d} \alpha} \log \Phi_{x}(\omega)\right)^{2}=\frac{1}{4}\left(\frac{\mathrm{d}}{\mathrm{d} \alpha} \log \left(\frac{\lambda-\alpha}{3 \lambda}\right)^{3}\right)^{2}=\frac{9}{4(\lambda-\alpha)^{2}}
$$

and hence via (19)

$$
\mathbf{E}\left\{\left|G\left(e^{j \omega}, \widehat{\beta}_{N}\right)-G\left(e^{j \omega}, \beta_{\circ}^{n}\right)\right|^{2}\right\} \approx \frac{3 \sigma^{2}}{N} \frac{\lambda}{(\lambda-\alpha)^{3}}\left[K_{n}(\omega)+\frac{3\left|G\left(e^{j \omega}, \beta_{\circ}\right)\right|^{2}}{\|G\|^{2}}\right] .
$$

Therefore, in this dead-zone non-linearity case, the input power $\Phi_{u}(\omega)=\lambda^{2} / 3$ should be made as large as possible. However, an interesting (and perhaps unexpected) phenomenon is that via (20) and using the same method which led to (32), then

$$
\operatorname{Var}\left\{\widehat{\alpha}_{N}\right\} \approx \frac{P_{\alpha}}{N}=\frac{\sigma^{2}}{N} \frac{4 \lambda}{(\lambda-\alpha)} \frac{1}{\|G\|^{2}} .
$$

Therefore, the variability of the estimate $\widehat{\alpha}_{N}$ of the non-linearity is subject to a 'noise floor', of

$$
\operatorname{Var}\left\{\widehat{\alpha}_{N}\right\}>\frac{4 \sigma^{2}}{N} \frac{1}{\|G\|^{2}} .
$$

Once exposed, this principle may be understood by the fact that the manifestation of the non-linearity on an input $\left|u_{t}\right|>\alpha$ is to transform it to $u_{t} \pm \alpha$ (depending upon the sign of the input). Therefore, the effect (of size $\alpha$ ) of the non-linearity is not magnified by using larger $u_{t}$, and hence its size relative to any measurement noise cannot be increased. This leads to the 'noise floor' which cannot be altered by choice of $\left\{u_{t}\right\}$.

Finally, when estimation involves a Hammerstein system consisting of a dead-zone in series with a linear system and a Pseudo-Random Binary Noise Sequence (PRBS) [10] is used as an input excitation, then the spectral densities required by (15)are supplied by Lemma 5.4.

Lemma 5.4. For $\left\{u_{t}\right\}$ i.i.d. with binomial distribution (30) and $X\left(u_{t}, \alpha\right)$ being the dead-zone nonlinearity (31) which is symmetric $\left(\alpha \triangleq \alpha_{1}=-\alpha_{2}\right)$ then for $\lambda>\alpha$

$$
\Phi_{x}(\omega)=(\lambda-\alpha)^{2}, \quad \Phi_{x x^{\prime}}(\omega)=\lambda-\alpha, \quad \Phi_{x^{\prime}}(\omega)=1 .
$$


Proof. The following equations may be derived by an examination of figure 4 .

$$
\begin{aligned}
\mathbf{E}\left\{x_{t}(\alpha)^{2}\right\} & =0.5 \times(\lambda-\alpha)^{2}+0.5 \times(\alpha-\lambda)^{2}=(\lambda-\alpha)^{2}, \\
\mathbf{E}\left\{x_{t}(\alpha) x_{t}^{\prime}(\alpha)\right\} & =0.5 \times(\lambda-\alpha) \times(-1)+0.5 \times(\alpha-\lambda) \times(1)=\lambda-\alpha, \\
\mathbf{E}\left\{\left[x_{t}^{\prime}(\alpha)\right]^{2}\right\} & =0.5 \times 1+0.5 \times 1=1 .
\end{aligned}
$$

Inserting the expressions given in Lemma 5.4 into the term $\|G\|_{x}$ of (15) yields

$$
\Phi_{x}(\omega) \Phi_{x^{\prime}}(\omega)-\left|\Phi_{x x^{\prime}}(\omega)\right|^{2}=(\lambda-\alpha)^{2}-(\lambda-\alpha)^{2}=0 .
$$

Thus the magnitude of the second term in (15) is infinite. As for the case of the saturation nonlinearity, this condition has arisen because the dead-zone non-linearity acts as a static gain of size $(\lambda-\alpha) / \lambda$ when subjected to a PRBS input.

\section{Simulation Examples}

Having derived the variance approximations (19),(20), it is of course important to test their accuracy. While some theoretical analysis involving convergence rates in the supporting Theorem 1 is possible by using the results in [19], this will not provide any hard bounds on the accuracy of (19) for finite model order $n$.

Therefore, in order to study this issue, experimental testing seems the only viable option. Consider then the motivational example provided in the introduction wherein the system (4) has its input-output response observed in the case where the output $\left\{y_{t}\right\}$ is corrupted by i.i.d. zero mean Gaussian noise of standard deviation $\sigma=0.001$, and with input $\left\{u_{t}\right\}$ being an i.i.d. zero mean and uniformly distributed process with $\sigma_{u}^{2}=\mathbf{E}\left\{u_{t}^{2}\right\}=1$. Consider further the situation introduced around figure 2 where a symmetric saturation of level $\alpha=0.5$ affects the input, and which is estimated together with a $25^{t h}$ order FIR model for the linear dynamics $G(q)$.

Then the true variability $\mathbf{E}\left\{\left|G\left(e^{j \omega}, \widehat{\beta}_{N}\right)-G\left(e^{j \omega}, \beta_{\circ}\right)\right|^{2}\right\}$ can be estimated via a Monte-Carlo simulation over different measurement noise and input realisations. This was done for 1000 such realisations each over $N=3000$ data points and the results are displayed in figure 5 with the ensuing sample variability shown as a solid line.

Also shown in that figure as a dash-dot line is the approximation (19) for this variability, calculated as (28). The agreement is excellent. The dashed line on the same figure is the approximation (3) that, as was discussed in the introduction, might at first glance be expected to be appropriate. However, as is clear from figure 5(a), this is not the case, and it is in fact vital to use the extra term that exists in (19); clearly the 'low-pass' effect invoked by the presence of the $\left|G\left(e^{j \omega}, \beta_{\circ}\right)\right|^{2}$ term dominates the approximation in such a way as to render it qualitatively as well as quantitatively "correct".

For the situation where $X\left(u_{t}, \alpha\right)$ is a symmetric dead-zone, with $\alpha=0.5$ then the corresponding Monte-Carlo simulation results are shown in figure 5(b). Again, excellent agreement between the Monte-Carlo estimate of the variability (solid line) and the new approximation (4) (formulated as (32)) is illustrated.

These examples provide encouraging empirical evidence for the utility of the approximation (19), but a key issue is that its derivation rests on $n$ being 'large' so that the underlying Fourier reconstructions have effectively converged.

To test the robustness of this dependence the previously described simulation involving a saturation non-linearity was repeated with the FIR model order reduced from 25 to 15 . The resulting comparison of the Monte-Carlo estimated variability of $G\left(e^{j \omega}, \widehat{\beta}_{N}\right)$ versus the approximation (19) is 
shown in figure 6(a). While the accuracy illustrated in figure 6(a) is somewhat degraded (relative to the higher order case profiled in figure 5(a)) the expression (19) is still a highly informative quantification of true variability, even for what may be considered a "low" model order.

To complete this simulation study section, some previous conclusions that were made on the basis of (19) are tested. Firstly, it was discussed that for the case of uniformly distributed input and saturation non-linearity then an optimal input power existed in the sense that (via the solution of (29)) it minimised the variability of $G\left(e^{j \omega}, \widehat{\beta}_{N}\right)$ at a specific frequency $\omega$.

The presence of this optimum is illustrated once again via Monte-Carlo simulation where, under the same conditions as previously reported for the saturation non-linearity case (and for $n=25$ ), the true variability of $G\left(e^{j \omega}, \widehat{\beta}_{N}\right)$ is estimated over 1000 realisations and at various input powers quantified by $\lambda$ (see (27)) and where $\Phi_{u}=\lambda^{2} / 3$. This is shown as the solid line in figure 6(b), and is profiled against the approximation (19) which in this case appears as (28). The presence of an optimal power around $\Phi_{u}=0.25$ is clear.

In the case of input dead-zone, it was discussed around (32) that another consequence of the approximation (20) was the indication that a 'noise floor' existed on $\operatorname{Var}\left\{\widehat{\alpha}_{N}\right\}$ in the sense that, no matter how large the input power excitation, a lower limit on $\operatorname{Var}\left\{\widehat{\alpha}_{N}\right\}$ existed and was given by (33). This lower limit is shown as the horizontal dash-dot line in figure 7(a). On that same figure, the 1000 run Monte-Carlo sample variance estimate of $\operatorname{Var}\left\{\widehat{\alpha}_{N}\right\}$ is shown as a solid line for various values of $\lambda$. The 'noise floor' phenomenon is clearly exposed.

Finally, it was discussed in $\S 4.3$ that (20) indicated that the achievable estimation accuracy for the non-linear component should be invariant to the dimension $n$ of the model used for the linear component. This phenomenon, for the dead-zone case just discussed, is illustrated in figure 7(b) where $P_{\alpha}$ is shown for a range of $n$. Clearly, once $n$ becomes large enough for the true system to be approximately in the model class (so that Theorem 1 and its ramifications apply), then as indicated by (20), further increases in $n$ have no impact on the variance $P_{\alpha}$.

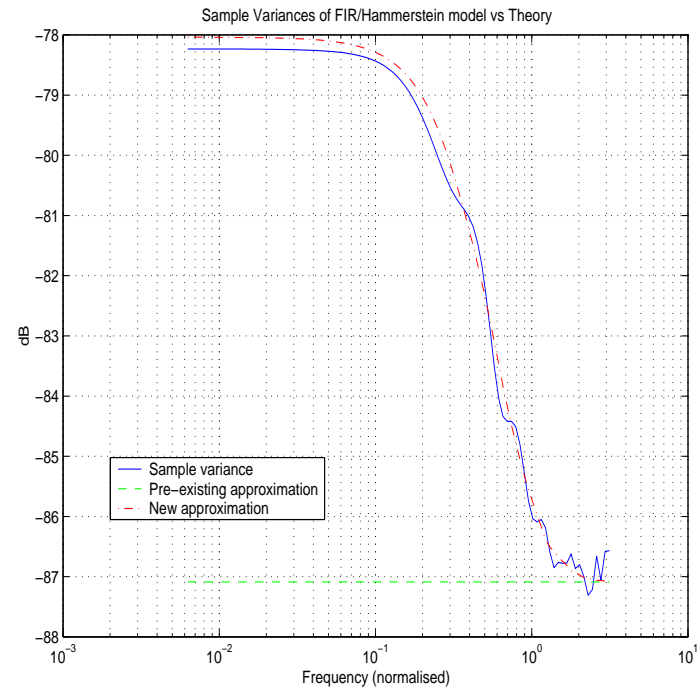

(a) Saturation Non-linearity

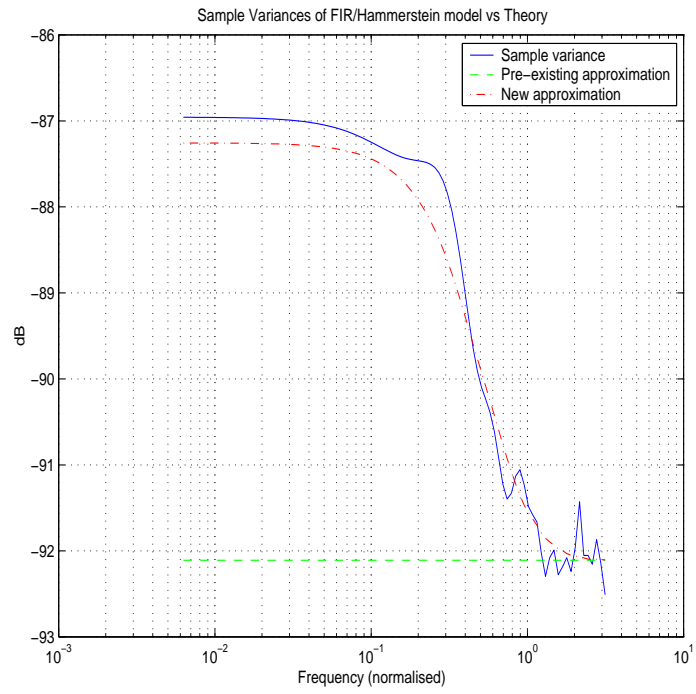

(b) Dead-zone Non-linearity

Figure 5: Variability of an $25^{\text {th }}$ order FIR estimate of the linear part of a Hammerstein system. Solid line is a Monte-Carlo estimate of true variability, dashed line is the pre-existing approximation (2) and the dash-dotted line is the new approximation (4). In the left hand figure, the case of a saturation non-linearity is shown, while the right hand figure illustrates the case of a dead-zone non-linearity 


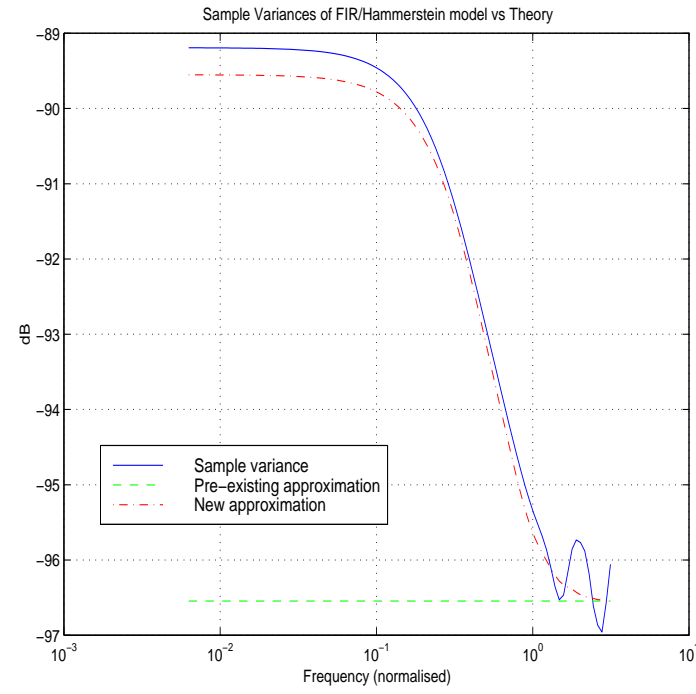

(a) Variability w.r.t. Frequency

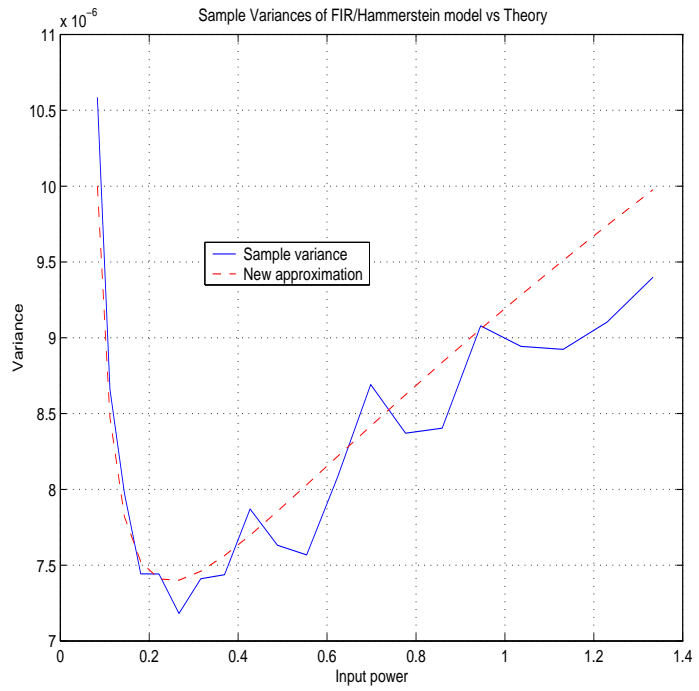

(b) Variability w.r.t. Input Power

Figure 6: Hammerstein System Estimate Variability, with the solid line being a Monte-Carlo estimate of true estimate variability and the non-linearity being of saturation type. In the left hand figure, the variability of a $15^{\text {th }}$ order FIR estimate of the linear model component is shown, with the dashed line the pre-existing approximation (2) and the dash-dotted line the new approximation (4). The right hand figure shows the variability of a $25^{\text {th }}$ order FIR estimate of the linear model component, but at a single frequency and for a range of input spectral powers. The dash-dotted line is the new approximation (28).

\section{To Model or to Bound?}

This paper began by considering the question of whether or not the inclusion of a non-linear component in a model structure was justified in terms of the tradeoff between decreasing bias error, but increasing variance error. Having now quantified the variance error via (19) some answer to this motivating issue is possible, but only once the bias error $G\left(e^{j \omega}\right)-G\left(e^{j \omega}, \beta_{\circ}\right)$ is clarified. Via (5)-(11), in the case where bias arises by ignoring a non-linearity in the true model, then the resultant estimation error will depend upon the spectral density $\Phi_{\varepsilon}$ of $\varepsilon_{t}(\beta)=G(q) x_{t}-G(q, \beta) u_{t}$ which may be expressed as

$$
\begin{aligned}
\Phi_{\varepsilon}(\omega) & =\left|G\left(e^{j \omega}\right)\right|^{2} \Phi_{x}(\omega)+\left|G\left(e^{j \omega}, \beta\right)\right|^{2} \Phi_{u}(\omega)-G\left(e^{j \omega}\right) \overline{G\left(e^{j \omega}, \beta\right) \Phi_{x u}(\omega)}-\overline{G\left(e^{j \omega}\right)} G\left(e^{j \omega}, \beta\right) \Phi_{x u}(\omega) \\
& =\Phi_{u}(\omega)\left[\left|G\left(e^{j \omega}, \beta\right)-G\left(e^{j \omega}\right) \frac{\Phi_{x u}(\omega)}{\Phi_{u}(\omega)}\right|^{2}+\left|G\left(e^{j \omega}\right)\right|^{2}\left(\frac{\Phi_{x}(\omega)}{\Phi_{u}(\omega)}-\left|\frac{\Phi_{x u}(\omega)}{\Phi_{u}(\omega)}\right|^{2}\right)\right] .
\end{aligned}
$$

Therefore, according to (9)-(11) and by Parseval's Theorem, the bias error can be characterised by representing $G\left(e^{j \omega}, \beta_{\circ}\right)$ as

$$
G\left(e^{j \omega}, \beta_{\circ}\right)=\underset{\beta \in \mathbf{R}^{n}}{\arg \min } \int_{-\pi}^{\pi}\left|G\left(e^{j \omega}, \beta\right)-G\left(e^{j \omega}\right) \frac{\Phi_{x u}(\omega)}{\Phi_{u}(\omega)}\right|^{2} \Phi_{u}(\omega) \mathrm{d} \omega .
$$

Clearly then (and as expected) whenever an input nonlinearity is present so that the cross-spectrum $\Phi_{x u}(\omega)$ is not equal to the auto-spectrum $\Phi_{u}(\omega)$, then bias error will be incurred. In general, it 


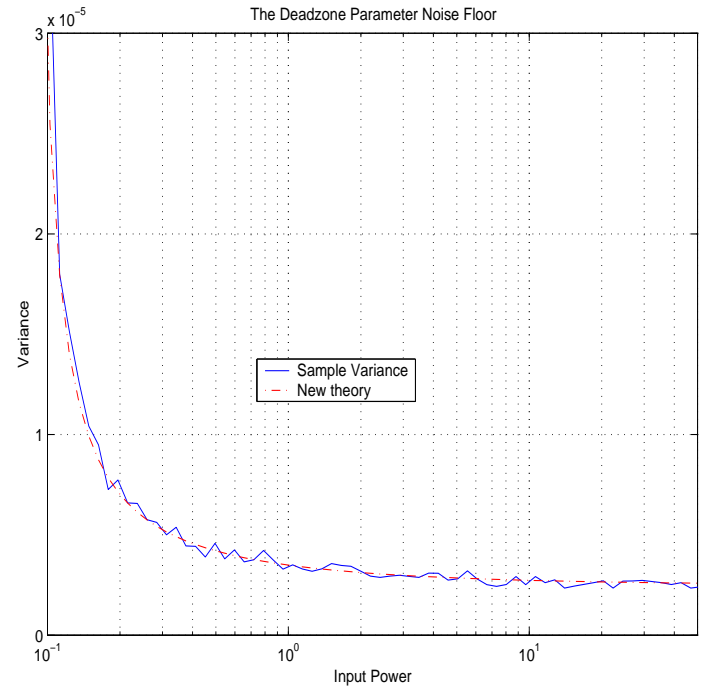

(a) $P_{\alpha}$ versus input power

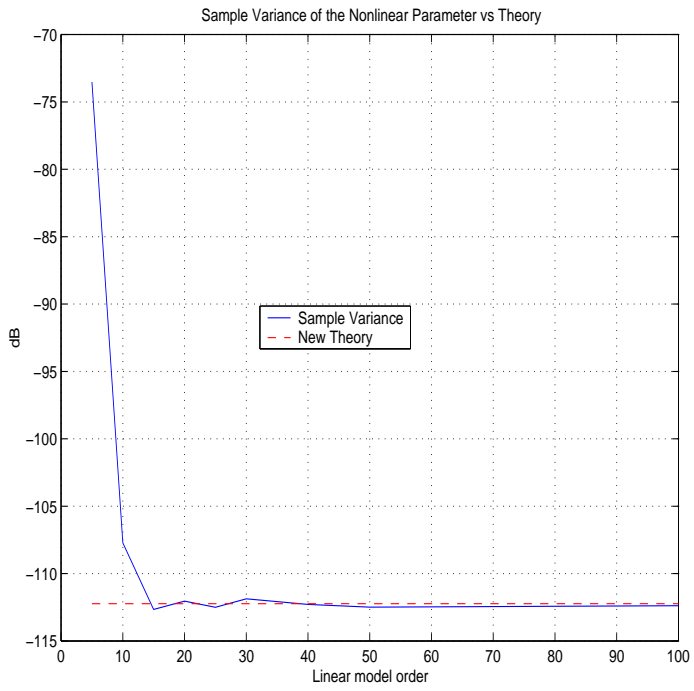

(b) $P_{\alpha}$ versus linear model order $n$

Figure 7: Variability $P_{\alpha}$ of the estimated (symmetric dead-zone) non-linear parameter of a Hammerstein system. Solid line is Monte-Carlo estimate of true variability and the dash-dotted line is the approximation (20). In the left-hand figure the case of dead-zone non-linearity and varying input power is considered. In the right hand figure a saturation non-linearity is used and the influence of linear system model order $n$ is shown.

is not possible to use (35) to exactly quantify the bias error. However, in the case in which $\left\{u_{t}\right\}$ is i.i.d. so that the spectral densities become constants it is evident from (35) that $G\left(e^{j \omega}, \beta_{\circ}\right)=$ $G\left(e^{j \omega}\right) \Phi_{x u}(\omega) / \Phi_{u}(\omega)$. Consequently, in this case, the increased bias error $\Delta_{b}$ incurred by choosing a model structure that ignores a static input non-linearity is

$$
\Delta_{b} \triangleq\left|G\left(e^{j \omega}\right)-G\left(e^{j \omega}, \beta_{\circ}\right)\right|^{2}=\left|G\left(e^{j \omega}\right)\right|^{2}\left(1-\frac{\Phi_{x u}(\omega)}{\Phi_{u}(\omega)}\right)^{2} .
$$

At the same time, it is evident from (20) that (in the same case of $\left\{u_{t}\right\}$ being i.i.d.) the increase $\Delta_{v}$ in variance error that is incurred by including a static non-linearity in the model structure is given by

$$
\Delta_{v}=\frac{\sigma^{2}}{4 N} \frac{\left|G\left(e^{j \omega}\right)\right|^{2}}{\|G\|^{2}}\left(\frac{\mathrm{d}}{\mathrm{d} \alpha} \log \Phi_{x}(\omega)\right)^{2}\left(\frac{\Phi_{x}(\omega)}{\Phi_{x}(\omega) \Phi_{x^{\prime}}(\omega)-\Phi_{x x^{\prime}}^{\star}(\omega) \Phi_{x x^{\prime}}(\omega)}\right) .
$$

The question of choosing a model structure that minimises the total estimation error then reduces to comparing the relative sizes of $\Delta_{b}$ and $\Delta_{v}$. In relation to this, notice firstly that because of the $1 / N$ factor in $\Delta_{v}$, then for long enough data lengths $\Delta_{v}$ will always be less than $\Delta_{b}$ regardless of other experimental conditions.

This provides an argument that generically, if in doubt, it is better to attempt the estimation of the non-linearity and suffer (perhaps unnecessarily) an increase $\Delta_{v}$ in estimation error, than it is to ignore the possibility of the non-linearity and risk the much larger bias error $\Delta_{b}$. Of course, if feasible, it is better again to use a model validation procedure in order to assess the presence of the non-linearity.

However, with this in mind, the key point made by the expressions $\Delta_{v}$ and $\Delta_{b}$ is that in no way is there any generic evidence to support a policy of ignoring non-linearities and bounding the resultant bias error as opposed to a strategy of including the non-linearity in the model structure and estimating it, and this answer directly addresses the motivating question posed in the introduction of this paper. 
For example, returning to the dead-zone non-linearity of $\S 5.2$ where the input $u_{t}$ is i.i.d. uniformly distributed in $[-\lambda, \lambda]$ and with symmetric dead-zone in $[-\alpha, \alpha]$, then using the values for $\Phi_{x}, \Phi_{x^{\prime}}, \Phi_{x x^{\prime}}$ and $\Phi_{u}$ calculated in $\S 5.2$ together with the following lemma

Lemma 7.1. For the case of the dead-zone non-linearity (31) which is symmetric in that $\alpha \triangleq \alpha_{1}=$ $-\alpha_{2}$ (see figure $\left.4(a)\right)$ and independent, uniformly distributed input $\left\{u_{t}\right\}$ according to (27) then for $\lambda>\alpha$

$$
\Phi_{x u}(\omega)=\frac{(\lambda-\alpha)^{2}(2 \lambda+\alpha)}{6 \lambda}
$$

Proof.

$$
p(x=A, u=B)=\left\{\begin{array}{cl}
\frac{1}{2 \lambda} & ; u \in[-\alpha, \alpha], x=0 \\
\frac{1}{2 \lambda} & ;|u|>\alpha, x=u \\
0 & ; \text { Otherwise }
\end{array}\right.
$$

Therefore

$$
\mathbf{E}\left\{x_{t} u_{t}\right\}=\frac{1}{2 \lambda} \int_{\alpha}^{\lambda} u(u-\alpha) \mathrm{d} u+\frac{1}{2 \lambda} \int_{-\lambda}^{-\alpha} u(u+\alpha) \mathrm{d} u=\frac{(\lambda-\alpha)^{2}(2 \lambda+\alpha)}{6 \lambda}
$$

allows the calculation

$$
\begin{gathered}
\Delta_{v}=\frac{\sigma^{2}}{N} \frac{\left|G\left(e^{j \omega}, \widehat{\beta}_{N}\right)\right|^{2}}{\|G\|^{2}} \frac{9 \lambda}{(\lambda-\alpha)^{3}} \\
\Delta_{b}=\frac{\alpha\left(3 \lambda^{2}-\alpha^{2}\right)}{2 \lambda^{3}}\left|G\left(e^{j \omega}\right)\right|^{2} .
\end{gathered}
$$

In this instance then, for a given degree of non-linearity as measured by the dead-zone limit $\alpha$, then (39) shows that the only factor that can decrease bias error $\Delta_{b}$ is a large input amplitude $\lambda$ (so that most input realisations are outside the deadzone), but by (38), this same strategy decreases the variance error $\Delta_{v}$ as well. Therefore, and as per the previous comments for the generic situation, it would seem difficult to encounter a situation in which $\Delta_{v}$ would not be smaller than $\Delta_{b}$, in which case estimation of the full non-linear structure is clearly most appropriate.

\section{Extensions to Other Model Structures}

The discussion to here has focussed on the 'FIR-like' class of model structures represented by (12) or an equivalent formulation such as (14). Clearly though, it is of interest to ascertain whether the quantifications (19), (20) (and hence also their implications) are applicable to other model structures as well. For example, consider the ARX model structure

$$
A(q) y_{t}=B(q) u_{t}+C(q) e_{t}
$$


where

$$
\begin{aligned}
& A(q) \triangleq a_{0}^{\prime}+a_{1}^{\prime} q+\cdots+a_{n-1}^{\prime} q^{n-1}+q^{n} \\
& B(q) \triangleq b_{0}^{\prime}+b_{1}^{\prime} q+\cdots+b_{n-1}^{\prime} q^{n-1} \\
& C(q) \triangleq \prod_{k=1}^{n}\left(q-\xi_{k}\right) .
\end{aligned}
$$

Here $C(q) / A(q)$ is a noise model with fixed zeros $\left\{\xi_{k}\right\}$, with the most common choice [10] being $\xi_{k}=0$.

This structure can be represented in an equivalent orthonormally parameterised form

$$
\left[1+\sum_{k=1}^{n} a_{k} \mathcal{B}_{k}(q)\right] y_{t}=\left[\sum_{k=1}^{n} b_{k} \mathcal{B}_{k}(q)\right] u_{t}+e_{t}
$$

where the $\left\{\mathcal{B}_{k}\right\}$ are as per (13) but with the poles $\left\{\xi_{k}\right\}$ being chosen as the zeros of $C(q)$. In turn, this provides a definition for the term $K_{n}(\omega)=\sum_{k=1}^{n}\left|\mathcal{B}_{k}\left(e^{j \omega}\right)\right|^{2}$ appearing in (19) so that the applicability of that approximation to the ARX case may be tested via Monte-Carlo simulation.

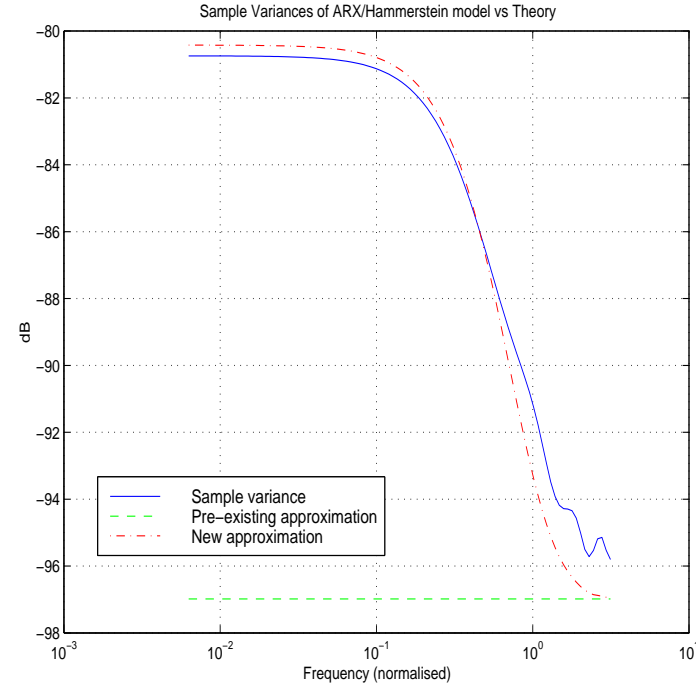

(a) Saturation Non-linearity

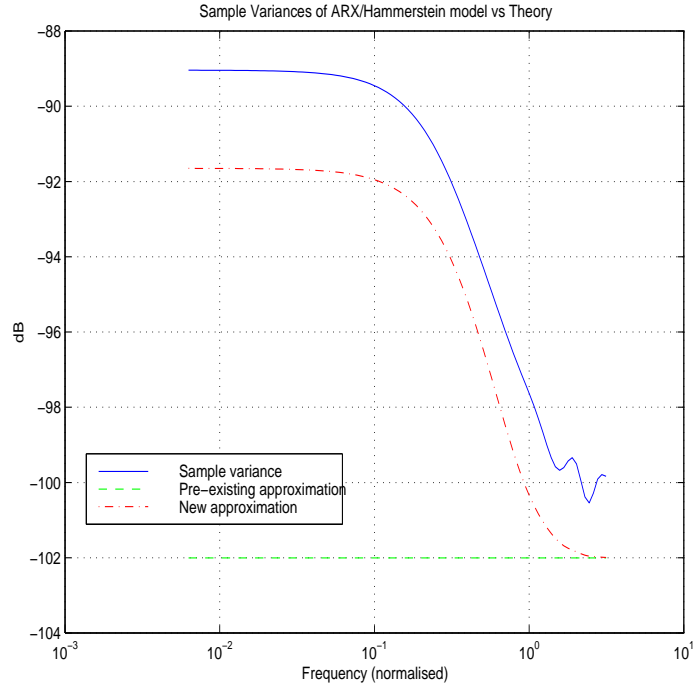

(b) Dead-zone Non-linearity

Figure 8: Variability of an $8^{\text {th }}$ order ARX model estimate of the linear part of a Hammerstein system. Solid line is Monte-Carlo estimate of true variability, dashed line is the pre-existing approximation (2) and the dash-dotted line is the new approximation (4). In the left hand figure, the case of a saturation non-linearity is shown, while the right hand figure illustrates the case of a dead-zone non-linearity

The results of such a test are shown in figure 8 . In that figure, as per the results presented in $\S 6$, the system (1) was used with the inputs, measurement noise levels and non-linearities being as detailed in $\S 6$ and with 1000 trials being used in the Monte-Carlo estimate of the true sample variability. Here the common choice $\xi_{k}=0$ was made so that $K_{n}(\omega)=n$.

The close agreement between the approximation (19) shown as the dash-dot line and the MonteCarlo estimate of true variability shown as the solid line indicates that indeed, (19) may be used in cases more general than the 'FIR-like' case for which it was derived via Theorem 1. 
To provide more detailed analysis as to why the approximation (19) is applicable to the ARX case when it was derived for the FIR-like case is beyond the scope of this paper but, as an indication of the issues involved, the reader is referred to [18].

\section{Conclusions}

The purpose of this paper has been to examine how the estimation accuracy of the linear portion of a Hammerstein model structure is affected by the mutual estimation of the non-linear component. This was addressed by deriving approximate expressions for the frequency domain variability of the linear and non-linear elements. Although these were established theoretically via Theorem 1 for FIR-like structures, they were shown via simulation to hold for ARX structures as well, and this indicates that the conclusions drawn from Theorem 1 will hold in a wider setting than just FIR modelling.

Although little emphasis has been given to the issue in the fore-going text, the central tools employed here are those of generalised Fourier and Toeplitz matrix analysis which have been developed in previous works $[19,18]$, but are extended here in the appendices to address certain novel issues that arise in the proof of Theorem 1. 


\section{A Proof of Theorem 1}

The key tools used in the proof of Theorem 1 are related to those presented in the earlier works [19, 18], but extended in Appendix B to provide certain multivariable generalisations that are required to accommodate the case when $\ell=\operatorname{dim}\{\alpha\}>1$. Also presented in Appendix B (Lemmas B.7 and B.6) are new results on the asymptotic algebraic properties of generalised Toeplitz matrices.

More specifically, the idea of a "generalised Toeplitz matrix" $M_{n}(F)$ which was introduced in [19] is extended here such that when $F$ is an arbitrary matrix-valued function on $[-\pi, \pi]$,

$$
M_{n}(F) \triangleq \frac{1}{2 \pi} \int_{-\pi}^{\pi} \Gamma_{n}\left(e^{j \omega}\right) \Gamma_{n}^{\star}\left(e^{j \omega}\right) \otimes F(\omega) \mathrm{d} \omega .
$$

where

$$
\Gamma_{n}(q) \triangleq\left[\mathcal{B}_{1}(q), \ldots, \mathcal{B}_{n}(q)\right]^{T}
$$

with the 'basis functions' $\mathcal{B}_{k}(q)$ being defined in (13).

Also, $\otimes$ is the Kronecker tensor product operator defined as follows. If $A$ is an $m \times n$-dimensional matrix such that $[A]_{k, \ell}=a_{k \ell}$ and $B$ is an $\ell \times p$-dimensional matrix then $A \otimes B$ is the $n \ell \times m p$ dimensional matrix given by

$$
A \otimes B \triangleq\left[\begin{array}{cccc}
a_{11} B & a_{12} B & \ldots & a_{1 n} B \\
a_{21} B & a_{22} B & \ldots & a_{2 n} B \\
\vdots & \vdots & & \vdots \\
a_{m 1} B & a_{m 2} B & \ldots & a_{m n} B
\end{array}\right]
$$

With this defined, the proof of Theorem 1 proceeds as follows.

Proof. Quantification of the variability of the estimate $\widehat{\theta}_{N}$ (around the value $\theta_{\circ}$ to which it is converging) may be achieved from the asymptotic distributional result $[10,13]$ (subscripts on square matrices indicate their dimension)

$$
\sqrt{N}\left(\widehat{\theta}_{N}-\theta_{\circ}\right) \stackrel{\mathcal{D}}{\rightarrow} \mathcal{N}\left(0, P_{m}\right)
$$

The covariance matrix $P_{m}$ in this expression is assumed to be positive definite and is defined in terms of two other matrices $R_{m}$ and $Q_{m}$ as

$$
P_{m} \triangleq R_{m}^{-1} Q_{m} R_{m}^{-1}
$$

which themselves are defined as

$$
\begin{aligned}
R_{m} & \triangleq \lim _{N \rightarrow \infty} \frac{\mathrm{d}^{2}}{\mathrm{~d} \theta \theta^{T}} \mathbf{E}\left\{V_{N}(\theta)\right\} \\
& =\lim _{N \rightarrow \infty} \frac{1}{N} \sum_{t=1}^{N}\left[\mathbf{E}\left\{\psi_{t}\left(\theta_{\circ}\right) \psi_{t}^{T}\left(\theta_{\circ}\right)\right\}-\mathbf{E}\left\{\varepsilon_{t}\left(\theta_{\circ}\right)\left(\frac{\mathrm{d} \psi_{t}(\theta)}{\mathrm{d} \theta}\right)^{T}\right\}\right] \\
Q_{m} & \triangleq \lim _{N \rightarrow \infty} \mathbf{E}\left\{\frac{\mathrm{d}}{\mathrm{d} \theta} V_{N}(\theta)\left(\frac{\mathrm{d}}{\mathrm{d} \theta} V_{N}(\theta)\right)^{T}\right\} \\
& =\lim _{N \rightarrow \infty} \frac{1}{N} \sum_{t=1}^{N} \sum_{\ell=1}^{N} \mathbf{E}\left\{\psi_{t}\left(\theta_{\circ}\right) \psi_{\ell}^{T}\left(\theta_{\circ}\right) \varepsilon_{t}\left(\theta_{\circ}\right) \varepsilon_{\ell}\left(\theta_{\circ}\right)\right\}
\end{aligned}
$$


where $\psi_{t}$ is the "predictor gradient" defined as

$$
\psi_{t}(\theta) \triangleq \frac{\mathrm{d} \widehat{y}_{t}(\theta)}{\mathrm{d} \theta} .
$$

Now, under the assumptions that the underlying true system is in the model class and that the data is such that the model structure is parameter identifiable, then $\varepsilon_{t}\left(\theta_{\circ}^{m}\right)=e_{t}$. This implies that

$$
\mathbf{E}\left\{\varepsilon_{t}\left(\theta_{\circ}^{m}\right)\left(\frac{\mathrm{d} \psi_{t}\left(\theta^{m}\right)}{\mathrm{d} \theta^{m}}\right)^{T}\right\}=\mathbf{E}\left\{e_{t}\left(\frac{\mathrm{d} \psi_{t}\left(\theta^{m}\right)}{\mathrm{d} \theta^{m}}\right)^{T}\right\}=\mathbf{E}\left\{e_{t}\right\} \mathbf{E}\left\{\left(\frac{\mathrm{d} \psi_{t}\left(\theta^{m}\right)}{\mathrm{d} \theta^{m}}\right)^{T}\right\}=0
$$

(this follows by the zero-meanness of $e_{t}$ which is independent of $\psi_{t}$, the latter depending only on past data) and hence

$$
R_{m} \triangleq \lim _{N \rightarrow \infty} \frac{1}{N} \sum_{t=1}^{N} \mathbf{E}\left\{\psi_{t}\left(\theta_{\circ}^{m}\right) \psi_{t}^{T}\left(\theta_{\circ}^{m}\right)\right\} .
$$

Similarly, again assuming that $\varepsilon_{t}\left(\theta_{\circ}^{m}\right)=e_{t}$, then

$Q_{m} \triangleq \lim _{N \rightarrow \infty} \frac{1}{N} \sum_{t=1}^{N} \sum_{\ell=1}^{N} \mathbf{E}\left\{\psi_{t}\left(\theta_{\circ}^{m}\right) \psi_{\ell}^{T}\left(\theta_{\circ}^{m}\right) e_{t} e_{\ell}\right\}=\sigma^{2} \lim _{N \rightarrow \infty} \frac{1}{N} \sum_{t=1}^{N} \mathbf{E}\left\{\psi_{t}\left(\theta_{\circ}^{m}\right) \psi_{t}^{T}\left(\theta_{\circ}^{m}\right)\right\}=\sigma^{2} R_{m}$

so that $P_{m}=\sigma^{2} R_{m}^{-1}$.

To proceed further, a key step is to express $P_{m}=\sigma^{2} R_{m}^{-1}$ via a frequency domain formulation. This may be achieved by noting that under the model structure (5), (12) for which the predictor (7) applies, then the predictor gradient $\psi_{t}\left(\theta^{m}\right)$ defined by (A.45) is given as

$$
\psi_{t}\left(\theta^{m}\right)=\left[\frac{\mathrm{d} G(q, \beta)}{\mathrm{d} \beta^{T}} x_{t}(\alpha), G(q, \beta) \frac{\mathrm{d} X\left(u_{t}, \alpha\right)}{\mathrm{d} \alpha^{T}}\right]^{T}=\left[\begin{array}{cc}
\Gamma_{n}\left(e^{j \omega}\right) & 0 \\
0 & G(q, \beta)
\end{array}\right]\left[\begin{array}{c}
x_{t} \\
x_{t}^{\prime}
\end{array}\right]
$$

where

$$
\Gamma_{n}(q) \triangleq\left[\mathcal{B}_{1}(q), \ldots, \mathcal{B}_{n}(q)\right]^{T}, \quad x_{t}^{\prime} \triangleq \frac{\mathrm{d}}{\mathrm{d} \alpha} X\left(u_{t}, \alpha\right)
$$

Therefore, under an assumption that $\left\{x_{t}\right\}$ and $\left\{x_{t}^{\prime}\right\}$ are jointly stationary [10] with associated (auto)spectral densities $\Phi_{x}(\omega)$ and $\Phi_{x^{\prime}}(\omega)$ and joint density $\Phi_{x x^{\prime}}(\omega)$, then via (A.46), $R_{m}$ may be represented as $\left(I_{\ell}\right.$ is the $\ell \times \ell$ identity matrix)

$$
\begin{aligned}
R_{m} & =\frac{1}{2 \pi} \int_{-\pi}^{\pi}\left[\begin{array}{cc}
\Gamma_{n}\left(e^{j \omega}\right) & 0 \\
0 & I_{\ell} G\left(e^{j \omega}, \beta_{\circ}^{n}\right)
\end{array}\right]\left[\begin{array}{cc}
\Phi_{x}(\omega) & \Phi_{x x^{\prime}}(\omega) \\
\Phi_{x x^{\prime}}^{\star}(\omega) & \Phi_{x^{\prime}}(\omega)
\end{array}\right]\left[\begin{array}{cc}
\Gamma_{n}^{\star}\left(e^{j \omega}\right) & 0 \\
0 & I_{\ell} \overline{G\left(e^{j \omega}, \beta_{\circ}^{n}\right)}
\end{array}\right] \mathrm{d} \omega \\
& =\frac{1}{2 \pi} \int_{-\pi}^{\pi}\left[\begin{array}{cc}
\Gamma_{n}\left(e^{j \omega}\right) \Phi_{x}(\omega) \Gamma_{n}^{\star}\left(e^{j \omega}\right) & \Gamma_{n}\left(e^{j \omega}\right) \Phi_{x x^{\prime}}(\omega) \overline{G\left(e^{j \omega}, \beta_{\circ}^{n}\right)} \\
G\left(e^{j \omega}, \beta_{\circ}^{n}\right) \Phi_{x x^{\prime}}^{\star}(\omega) \Gamma_{n}^{\star}\left(e^{j \omega}\right) & \left|G\left(e^{j \omega}, \beta_{\circ}^{n}\right)\right|^{2} \Phi_{x^{\prime}}(\omega)
\end{array}\right] \mathrm{d} \omega .
\end{aligned}
$$

However, using the generalised Toeplitz matrix definition (A.40), then $R_{m}$ is expressible in partitioned form as

$$
R_{m}=\left[\begin{array}{cc}
M_{n}\left(\Phi_{x}\right) & M_{n}\left(\Phi_{x x^{\prime}}\right) \widetilde{\beta}_{\circ} \\
\widetilde{\beta}_{\circ}^{T} M_{n}\left(\Phi_{x x^{\prime}}^{\star}\right) & \widetilde{\beta}_{\circ}^{T} M_{n}\left(\Phi_{x^{\prime}}\right) \widetilde{\beta}_{\circ}
\end{array}\right]
$$

where $\widetilde{\beta}_{\circ} \triangleq \beta_{\circ}^{n} \otimes I_{\ell}$. Now, the convergence result (A.41) yields

$$
\sqrt{N}\left(\widehat{\beta}_{N}-\beta_{\circ}^{n}\right) \stackrel{\mathcal{D}}{\rightarrow} \mathcal{N}\left(0, P_{\beta}\right) \text { as } N \rightarrow \infty,
$$


where $P_{\beta}$ is defined via

$$
P_{m}=\sigma^{2} R_{m}^{-1}=\left[\begin{array}{cc}
P_{\beta} & P_{\beta \alpha^{T}} \\
P_{\alpha \beta^{T}} & P_{\alpha}
\end{array}\right]
$$

Furthermore, since

$$
G\left(q, \widehat{\beta}_{N}\right)-G\left(q, \beta_{\circ}^{n}\right)=\Gamma_{n}^{T}(q)\left(\widehat{\beta}_{N}-\beta_{\circ}^{n}\right),
$$

then the variance error in the estimate $G\left(q, \widehat{\beta}_{N}\right)$ of the linear dynamics $G(q)$ may be quantified as

$$
\lim _{N \rightarrow \infty} N \mathbf{E}\left\{\left|G\left(e^{j \omega}, \widehat{\beta}_{N}\right)-G\left(e^{j \omega}, \beta_{\circ}^{n}\right)\right|^{2}\right\}=\Gamma_{n}^{\star}\left(e^{j \omega}\right) P_{\beta} \Gamma_{n}\left(e^{j \omega}\right) .
$$

Furthermore, using the formula for the inverse of a partitioned matrix [3, 29] and the matrix inversion lemma [4],

$$
\lim _{N \rightarrow \infty} N \operatorname{Cov}\left\{\widehat{\alpha}_{N}\right\}=P_{\alpha} \triangleq \sigma^{2}\left[\widetilde{\beta}_{\circ}^{T} M_{n}\left(\Phi_{x^{\prime}}\right) \widetilde{\beta}_{\circ}-\widetilde{\beta}_{\circ}^{T} M_{n}\left(\Phi_{x^{\prime} x}\right) M_{n}^{-1}\left(\Phi_{x}\right) M_{n}\left(\Phi_{x x^{\prime}}\right) \widetilde{\beta}_{\circ}\right]^{-1}
$$

Similarly

$$
\begin{aligned}
\lim _{N \rightarrow \infty} N \operatorname{Cov}\left\{\widehat{\beta}_{N}\right\}=P_{\beta} & =\sigma^{2}\left[M_{n}\left(\Phi_{x}\right)-M_{n}\left(\Phi_{x x^{\prime}}\right) \widetilde{\beta}_{\circ}\left[\widetilde{\beta}_{\circ}^{T} M_{n}\left(\Phi_{x^{\prime}}\right) \widetilde{\beta}_{\circ}\right]^{-1} \widetilde{\beta}_{\circ}^{T} M_{n}\left(\Phi_{x x^{\prime}}^{\star}\right)\right]^{-1} \\
& =\sigma^{2} M_{n}^{-1}\left(\Phi_{x}\right)+M_{n}^{-1}\left(\Phi_{x}\right) \Omega_{n}(\omega) M_{n}^{-1}\left(\Phi_{x}\right)
\end{aligned}
$$

where

$$
\Omega_{n}(\omega) \triangleq M_{n}\left(\Phi_{x x^{\prime}}\right) \widetilde{\beta}_{\circ} P_{\alpha} \widetilde{\beta}_{\circ}^{T} M_{n}\left(\Phi_{x x^{\prime}}^{\star}\right)
$$

Substituting this into (A.50) provides

$$
\begin{aligned}
\frac{N}{K_{n}(\omega)} \mathbf{E}\left\{\left|G\left(e^{j \omega}, \widehat{\beta}_{N}\right)-G\left(e^{j \omega}, \beta_{\circ}^{n}\right)\right|^{2}\right\} & =\frac{\sigma^{2}}{K_{n}(\omega)} \Gamma_{n}^{\star}\left(e^{j \omega}\right) M_{n}^{-1}\left(\Phi_{x}\right) \Gamma_{n}\left(e^{j \omega}\right) \\
& +\frac{\sigma^{2}}{K_{n}(\omega)} \Gamma_{n}^{\star}\left(e^{j \omega}\right) M_{n}^{-1}\left(\Phi_{x}\right) \Omega_{n}(\omega) M_{n}^{-1}\left(\Phi_{x}\right) \Gamma_{n}\left(e^{j \omega}\right)
\end{aligned}
$$

where

$$
K_{n}(\omega) \triangleq \sum_{k=1}^{n}\left|\mathcal{B}_{k}\left(e^{j \omega}\right)\right|^{2} .
$$

The point of the normalisation by $K_{n}(\omega)$ is that via the results of [19], (see Theorem B.1),

$$
\lim _{n \rightarrow \infty} \frac{\sigma^{2}}{K_{n}(\omega)} \Gamma_{n}^{\star}\left(e^{j \omega}\right) M_{n}^{-1}\left(\Phi_{x}\right) \Gamma_{n}\left(e^{j \omega}\right)=\frac{\sigma^{2}}{\Phi_{x}(\omega)}
$$

which yields the first term in (15). The second term in (A.51) may be dealt with by noting that

$$
\begin{aligned}
& \leq\left|\frac{\Gamma_{n}^{\star}\left(e^{j \omega}\right)\left[M_{n}^{-1}\left(\Phi_{x}\right) \Omega_{n}(\omega) M_{n}^{-1}\left(\Phi_{x}\right)-M_{n}\left(1 / \Phi_{x}\right) \Omega_{n}(\omega) M_{n}\left(1 / \Phi_{x}\right)\right] \Gamma_{n}\left(e^{j \omega}\right)}{K_{n}(\omega)}\right| \\
& \left|\frac{\Gamma_{n}^{\star}\left(e^{j \omega}\right)\left[M_{n}^{-1}\left(\Phi_{x}\right)-M_{n}\left(1 / \Phi_{x}\right)\right] \Omega_{n}(\omega) M_{n}^{-1}\left(\Phi_{x}\right) \Gamma_{n}\left(e^{j \omega}\right)}{K_{n}(\omega)}\right|+ \\
& \frac{\Gamma_{n}^{\star}\left(e^{j \omega}\right) M_{n}\left(1 / \Phi_{x}\right) \Omega_{n}(\omega)\left[M_{n}\left(1 / \Phi_{x}\right)-M_{n}^{-1}\left(\Phi_{x}\right)\right] \Gamma_{n}\left(e^{j \omega}\right)}{K_{n}(\omega)} \mid
\end{aligned}
$$


The first term in (A.53) above can be bounded using the Cauchy-Schwarz inequality as

$$
\begin{aligned}
& \left|\frac{\Gamma_{n}^{\star}\left(e^{j \omega}\right)\left[M_{n}^{-1}\left(\Phi_{x}\right)-M_{n}\left(1 / \Phi_{x}\right)\right] \Omega_{n}(\omega) M_{n}^{-1}\left(\Phi_{x}\right) \Gamma_{n}\left(e^{j \omega}\right)}{K_{n}(\omega)}\right|^{2} \\
\leq & \frac{\Gamma_{n}^{\star}\left(e^{j \omega}\right)\left[M_{n}^{-1}\left(\Phi_{x}\right)-M_{n}\left(1 / \Phi_{x}\right)\right]^{2} \Gamma_{n}\left(e^{j \omega}\right)}{K_{n}(\omega)} \times \frac{\Gamma_{n}^{\star}\left(e^{j \omega}\right) M_{n}^{-1}\left(\Phi_{x}\right) \Omega_{n}^{2}(\omega) M_{n}^{-1}\left(\Phi_{x}\right) \Gamma_{n}\left(e^{j \omega}\right)}{K_{n}(\omega)} .
\end{aligned}
$$

However, via Theorem B.1

$$
\lim _{n \rightarrow \infty} \frac{\Gamma_{n}^{\star}\left(e^{j \omega}\right)\left[M_{n}^{-1}\left(\Phi_{x}\right)-M_{n}\left(1 / \Phi_{x}\right)\right]^{2} \Gamma_{n}\left(e^{j \omega}\right)}{K_{n}(\omega)}=0 .
$$

In addition, since $K_{n}(\omega)=\Gamma_{n}^{\star}\left(e^{j \omega}\right) \Gamma_{n}\left(e^{j \omega}\right)$ and [19] $\left\|M_{n}(f)\right\|_{2} \leq \max _{\omega \in[-\pi, \pi]}|f(\omega)|$ then by the assumption of parameter identifiability which implies that $\left\|P_{\alpha}\right\|_{2}<\infty$ and hence that $\left\|\Omega_{n}\right\|_{2}<\infty$

$$
\begin{aligned}
\frac{\Gamma_{n}^{\star}\left(e^{j \omega}\right) M_{n}^{-1}\left(\Phi_{x}\right) \Omega_{n}^{2}(\omega) M_{n}^{-1}\left(\Phi_{x}\right) \Gamma_{n}\left(e^{j \omega}\right)}{K_{n}(\omega)} & \leq\left\|\Omega_{n}\right\|_{2}^{2}\left\|M_{n}^{-1}\left(\Phi_{x}\right)\right\|_{2}^{2} \\
& \leq\left\|\Omega_{n}\right\|_{2}^{2} \max _{\omega \in[-\pi, \pi]} \frac{1}{\Phi_{x}^{2}(\omega)} \\
& <\infty
\end{aligned}
$$

and therefore the first term in (A.53) converges to zero as $n \rightarrow \infty$ and $\forall \omega \in[-\pi, \pi]$. The second term of (A.53) can then be shown to tend to zero as $n \rightarrow \infty$ in a similar manner. Therefore using these developments together with two successive applications of Lemma B.2,

$$
\begin{aligned}
& \lim _{n \rightarrow \infty} \frac{1}{K_{n}(\omega)} \Gamma_{n}^{\star}\left(e^{j \omega}\right) M_{n}^{-1}\left(\Phi_{x}\right) \Omega_{n}(\omega) M_{n}^{-1}\left(\Phi_{x}\right) \Gamma_{n}\left(e^{j \omega}\right) \\
= & \lim _{n \rightarrow \infty} \frac{1}{K_{n}(\omega)} \Gamma_{n}^{\star}\left(e^{j \omega}\right) M_{n}\left(1 / \Phi_{x}\right) \Omega_{n}(\omega) M_{n}\left(1 / \Phi_{x}\right) \Gamma_{n}\left(e^{j \omega}\right) \\
= & \frac{1}{\Phi_{x}^{2}(\omega)} \lim _{n \rightarrow \infty} \frac{1}{K_{n}(\omega)} \Gamma_{n}^{\star}\left(e^{j \omega}\right) M_{n}\left(\Phi_{x x^{\prime}}\right) \widetilde{\beta}_{\circ} P_{\alpha} \widetilde{\beta}_{\circ}^{T} M_{n}\left(\Phi_{x x^{\prime}}^{\star} \Gamma_{n}\left(e^{j \omega}\right)\right. \\
= & \frac{1}{\Phi_{x}^{2}(\omega)} \Phi_{x x^{\prime}}(\omega) P_{\alpha} \Phi_{x x^{\prime}}^{\star}(\omega) \times \lim _{n \rightarrow \infty} \frac{\left|G\left(e^{j \omega}, \beta_{\circ}^{n}\right)\right|^{2}}{K_{n}(\omega)} \\
= & \frac{1}{4}\left(\frac{\mathrm{d}}{\mathrm{d} \alpha} \log \Phi_{x}(\omega)\right)^{T} P_{\alpha}\left(\frac{\mathrm{d}}{\mathrm{d} \alpha} \log \Phi_{x}(\omega)\right) \times \lim _{n \rightarrow \infty} \frac{\left|G\left(e^{j \omega}, \beta_{\circ}^{n}\right)\right|^{2}}{K_{n}(\omega)} .
\end{aligned}
$$

In progressing to the last line, we have used the fact that

$$
\begin{aligned}
\Phi_{x^{\prime} x}(\omega) & =\sum_{\tau=-\infty}^{\infty} \mathbf{E}\left\{x_{t}(\alpha) \frac{\mathrm{d}}{\mathrm{d} \alpha} x_{t+\tau}(\alpha)\right\} e^{-j \omega \tau} \\
& =\frac{1}{2} \frac{\mathrm{d}}{\mathrm{d} \alpha} \sum_{\tau=-\infty}^{\infty} \mathbf{E}\left\{x_{t}(\alpha) x_{t+\tau}(\alpha)\right\} e^{-j \omega \tau} \\
& =\frac{1}{2} \frac{\mathrm{d}}{\mathrm{d} \alpha} \Phi_{x}(\omega, \alpha)=\frac{1}{2} \Phi_{x}(\omega) \frac{\mathrm{d}}{\mathrm{d} \alpha} \log \Phi_{x}(\omega) .
\end{aligned}
$$

This establishes (15). To prove (17), note that via Lemma B.6,

$$
\lim _{n \rightarrow \infty}\left\|M_{n}(F) M_{n}(H)-M_{n}(F H)\right\|=0,
$$


where the norm is a special one defined there. Applying this principle then leads to the approximation

$$
\begin{aligned}
\lim _{n \rightarrow \infty} \frac{P_{\alpha}}{\sigma^{2}} & =\lim _{n \rightarrow \infty}\left[\widetilde{\beta}_{\circ}^{T} M_{n}\left(\Phi_{x^{\prime}}\right) \widetilde{\beta}_{\circ}-\widetilde{\beta}_{\circ}^{T} M_{n}\left(\Phi_{x x^{\prime}}^{\star}\right) M_{n}^{-1}\left(\Phi_{x}\right) M_{n}\left(\Phi_{x x^{\prime}}\right) \widetilde{\beta}_{\circ}\right]^{-1} \\
& =\lim _{n \rightarrow \infty}\left[\widetilde{\beta}_{\circ}^{T} M_{n}\left(\Phi_{x^{\prime}}-\frac{\Phi_{x x^{\prime}}^{\star} \Phi_{x x^{\prime}}}{\Phi_{x}}\right) \widetilde{\beta}_{\circ}\right]^{-1} \\
& =\lim _{n \rightarrow \infty}\left[\frac{1}{2 \pi} \int_{-\pi}^{\pi}\left|G\left(e^{j \omega}, \beta_{\circ}^{n}\right)\right|^{2}\left(\frac{\Phi_{x}(\omega) \Phi_{x^{\prime}}(\omega)-\Phi_{x x^{\prime}}^{\star}(\omega) \Phi_{x x^{\prime}}(\omega)}{\Phi_{x}(\omega)}\right) \mathrm{d} \omega\right]^{-1}
\end{aligned}
$$




\section{B Technical Lemmata}

Theorem B.1. Suppose that the scalar valued function $f(\omega)$ is Lipschitz continuous of some order $\eta>0$ and that in the definition of the $\left\{\mathcal{B}_{k}\right\}$ forming the $\Gamma_{n}$ (and hence $\left.M_{n}(\cdot)\right)$ that the poles $\left\{\xi_{k}\right\}$ satisfy $\left|\xi_{k}\right| \leq 1-\delta$ for some $\delta>0$. Then for any integer $k$ (possibly negative)

$$
\lim _{n \rightarrow \infty} \frac{1}{K_{n}(\omega)} \Gamma_{n}^{\star}\left(e^{j \omega}\right) M_{n}^{-k}(f) \Gamma_{n}\left(e^{j \lambda}\right)= \begin{cases}f^{-k}(\omega) & ; \omega=\lambda \\ 0 & ; \omega \neq \lambda\end{cases}
$$

componentwise and uniformly on $\omega \in[-\pi, \pi]$.

Proof. See Corollary 5.3 of [19].

Lemma B.2. Let $F(\omega) \in \mathbf{C}^{1 \times \ell}$ be a row vector valued function which is componentwise Lipschitz continuous of some order $\eta>0$, and let $Q \in \mathbf{R}^{\ell n \times \ell n}$ be a symmetric, positive definite matrix with $\|Q\|_{2}<\infty$ for all $n$. Suppose also that in the definition of the $\left\{\mathcal{B}_{k}\right\}$ forming the $\Gamma_{n}$ (and hence $\left.M_{n}(\cdot)\right)$ that the poles $\left\{\xi_{k}\right\}$ satisfy $\left|\xi_{k}\right| \leq 1-\delta$ for some $\delta>0$. Then for all $\omega \in[-\pi, \pi]$

$$
\lim _{n \rightarrow \infty} \frac{1}{K_{n}(\omega)} \Gamma_{n}^{\star}\left(e^{j \omega}\right) M_{n}(F) Q M_{n}\left(F^{T}\right) \Gamma_{n}\left(e^{j \omega}\right)=F(\omega) \lim _{n \rightarrow \infty} \frac{1}{K_{n}(\omega)} \widetilde{\Gamma}_{n}^{\star}\left(e^{j \omega}\right) Q \widetilde{\Gamma}_{n}\left(e^{j \omega}\right) F^{T}(\omega),
$$

where

$$
K_{n}(\omega) \triangleq \sum_{k=1}^{n}\left|\mathcal{B}_{k}\left(e^{j \omega}\right)\right|^{2}=\sum_{k=1}^{n} \frac{1-\left|\xi_{k}\right|^{2}}{\left|e^{j \omega}-\xi_{k}\right|^{2}}, \quad \widetilde{\Gamma}_{n}\left(e^{j \omega}\right) \triangleq \Gamma_{n}\left(e^{j \omega}\right) \otimes I_{\ell}
$$

Proof. Begin by defining $\widetilde{F}(\omega)$ and $L$ as

$$
\widetilde{F} \triangleq \operatorname{diag}(F), \quad L \triangleq \operatorname{ones}(\ell, 1) \triangleq[1,1, \ldots, 1]^{T} .
$$

and further define for a fixed $\omega \in[-\pi, \pi]$ a function $\widetilde{G}(\sigma) \triangleq \widetilde{F}(\sigma)-\widetilde{F}(\omega)$ of $\sigma$. Now by Lemma B.3

$$
\Gamma_{n}^{\star}\left(e^{j \omega}\right) M_{n}(F) Q M_{n}\left(F^{T}\right) \Gamma_{n}\left(e^{j \omega}\right)=L^{T} \widetilde{\Gamma}_{n}^{\star}\left(e^{j \omega}\right) M_{n}(\widetilde{F}) Q M_{n}(\widetilde{F}) \widetilde{\Gamma}_{n}\left(e^{j \omega}\right) L .
$$

Therefore, since $L^{T} \widetilde{\Gamma}_{n}^{\star}\left(e^{j \omega}\right)\left[I_{n} \otimes \widetilde{F}\right]=F \widetilde{\Gamma}_{n}^{\star}\left(e^{j \omega}\right)$ then

$$
\begin{aligned}
& \frac{1}{K_{n}(\omega)}\left|\Gamma_{n}^{\star}\left(e^{j \omega}\right) M_{n}(F) Q M_{n}\left(F^{T}\right) \Gamma_{n}\left(e^{j \omega}\right)-F(\omega) \widetilde{\Gamma}_{n}^{\star}\left(e^{j \omega}\right) Q \widetilde{\Gamma}_{n}\left(e^{j \omega}\right) F^{T}(\omega)\right| \\
= & \frac{1}{K_{n}(\omega)}\left|L^{T} \widetilde{\Gamma}_{n}^{\star}\left(e^{j \omega}\right) M_{n}(\widetilde{F}) Q M_{n}(\widetilde{F}) \widetilde{\Gamma}_{n}\left(e^{j \omega}\right) L-F(\omega) \widetilde{\Gamma}_{n}^{\star}\left(e^{j \omega}\right) Q \widetilde{\Gamma}_{n}\left(e^{j \omega}\right) F^{T}(\omega)\right| \\
\leq & \frac{1}{K_{n}(\omega)}\left|L^{T} \widetilde{\Gamma}_{n}^{\star}\left(e^{j \omega}\right) M_{n}(\widetilde{G}) Q M_{n}(\widetilde{G}) \widetilde{\Gamma}_{n}\left(e^{j \omega}\right) L\right|+\frac{2}{K_{n}(\omega)}\left|L^{T} \widetilde{\Gamma}_{n}^{\star}\left(e^{j \omega}\right) M_{n}(\widetilde{G}) Q \widetilde{\Gamma}_{n}\left(e^{j \omega}\right) F^{T}(\omega)\right| \\
= & \frac{1}{K_{n}(\omega)}\left|L^{T} \widetilde{\Gamma}_{n}^{\star}\left(e^{j \omega}\right) M_{n}(\widetilde{G}) Q M_{n}(\widetilde{G}) \widetilde{\Gamma}_{n}\left(e^{j \omega}\right) L\right|+\frac{2}{K_{n}(\omega)}\left|\Gamma_{n}^{\star}\left(e^{j \omega}\right) M_{n}(G) Q \widetilde{\Gamma}_{n}\left(e^{j \omega}\right) F^{T}(\omega)\right|
\end{aligned}
$$

where the last line follows by Lemma B.3. However, by Lemma B.4 and since $G(\omega)=0$ while $\|Q\|_{2}<\infty$

$$
\lim _{n \rightarrow \infty} \frac{1}{K_{n}(\omega)} \Gamma_{n}^{\star}\left(e^{j \omega}\right) M_{n}(G) Q \widetilde{\Gamma}_{n}\left(e^{j \omega}\right)=G(\omega) \lim _{n \rightarrow \infty} \frac{1}{K_{n}(\omega)} \widetilde{\Gamma}_{n}^{\star}\left(e^{j \omega}\right) Q \widetilde{\Gamma}_{n}\left(e^{j \omega}\right)=0 .
$$


Furthermore, by the Cauchy-Schwarz inequality and Lemma B.3

$$
\begin{aligned}
& \lim _{n \rightarrow \infty} \frac{1}{K_{n}(\omega)}\left|L^{T} \widetilde{\Gamma}_{n}^{\star}\left(e^{j \omega}\right) M_{n}(\widetilde{G}) Q M_{n}(\widetilde{G}) \widetilde{\Gamma}_{n}\left(e^{j \omega}\right) L\right| \\
\leq & \|Q\|_{2} \lim _{n \rightarrow \infty} \frac{1}{K_{n}(\omega)}\left|L^{T} \widetilde{\Gamma}_{n}^{\star}\left(e^{j \omega}\right) M_{n}(\widetilde{G}) M_{n}(\widetilde{G}) \widetilde{\Gamma}_{n}\left(e^{j \omega}\right) L\right| \\
= & \|Q\|_{2} \lim _{n \rightarrow \infty} \frac{1}{K_{n}(\omega)}\left|\Gamma_{n}^{\star}\left(e^{j \omega}\right) M_{n}(G) M_{n}\left(G^{T}\right) \Gamma_{n}\left(e^{j \omega}\right)\right| . \\
\leq & \|Q\|_{2} \sum_{r=1}^{\ell}\left|\frac{\Gamma_{n}^{\star}\left(e^{j \omega}\right) M_{n}^{2}\left([G]_{r}\right) \Gamma_{n}\left(e^{j \omega}\right)}{K_{n}(\omega)}\right|
\end{aligned}
$$

Finally, by Lemma B.1

$$
\lim _{n \rightarrow \infty} \frac{\Gamma_{n}^{\star}\left(e^{j \omega}\right) M_{n}^{2}\left([G]_{r}\right) \Gamma_{n}\left(e^{j \omega}\right)}{K_{n}(\omega)}=[G(\omega)]_{r}^{2}=0 .
$$

Lemma B.3. Let $F(\omega) \in \mathbf{C}^{1 \times \ell}$ be a row-vector valued function. Then

$$
\Gamma_{n}^{\star}\left(e^{j \omega}\right) M_{n}(F)=L^{T}\left[\Gamma_{n}\left(e^{j \omega}\right) \otimes I_{\ell}\right] M_{n}(\widetilde{F}),
$$

where

$$
\widetilde{F} \triangleq \operatorname{diag}(F), \quad L \triangleq \operatorname{ones}(\ell, 1) \triangleq[1,1, \ldots, 1]^{T}
$$

Proof.

$$
\begin{aligned}
L^{T}\left[\Gamma_{n}\left(e^{j \omega}\right) \otimes I_{\ell}\right] M_{n}(\widetilde{F}) & =L^{T} \frac{1}{2 \pi} \int_{-\pi}^{\pi}\left[\Gamma_{n}^{\star}\left(e^{j \omega}\right) \otimes I_{\ell}\right]\left[\Gamma_{n}\left(e^{j \sigma}\right) \Gamma_{n}^{\star}\left(e^{j \sigma}\right) \otimes \widetilde{F}(\sigma)\right] \mathrm{d} \sigma \\
& =\frac{1}{2 \pi} \int_{-\pi}^{\pi}\left[\Gamma_{n}^{\star}\left(e^{j \omega}\right) \otimes L^{T}\right]\left[\Gamma_{n}\left(e^{j \sigma}\right) \Gamma_{n}^{\star}\left(e^{j \sigma}\right) \otimes \widetilde{F}(\sigma)\right] \mathrm{d} \sigma \\
& =\frac{1}{2 \pi} \int_{-\pi}^{\pi}\left[\Gamma_{n}^{\star}\left(e^{j \omega}\right) \Gamma_{n}\left(e^{j \sigma}\right) \Gamma_{n}^{\star}\left(e^{j \sigma}\right)\right] \otimes\left[L^{T} \widetilde{F}(\sigma)\right] \mathrm{d} \sigma \\
& =\Gamma_{n}^{\star}\left(e^{j \omega}\right)\left[\frac{1}{2 \pi} \int_{\pi}^{\pi} \Gamma_{n}\left(e^{j \sigma}\right) \Gamma_{n}^{\star}\left(e^{j \sigma}\right) \otimes F(\sigma) \mathrm{d} \sigma\right] \\
& =\Gamma_{n}^{\star}\left(e^{j \omega}\right) M_{n}(F) .
\end{aligned}
$$

Lemma B.4. Let $F(\omega) \in \mathbf{C}^{1 \times \ell}$ be a row vector valued function which is componentwise Lipschitz continuous of some order $\eta>0$, and let $Q \in \mathbf{R}^{\ell n \times \ell n}$ be a symmetric, positive definite matrix with $\|Q\|_{2}<\infty$. Suppose also that in the definition of the $\left\{\mathcal{B}_{k}\right\}$ forming the $\Gamma_{n}$ (and hence $\left.M_{n}(\cdot)\right)$ that the poles $\left\{\xi_{k}\right\}$ satisfy $\left|\xi_{k}\right| \leq 1-\delta$ for some $\delta>0$. Then for all $\omega \in[-\pi, \pi]$ and denoting by $[\cdot]_{k}$ the $k$ 'th element of a vector

$$
\left[\lim _{n \rightarrow \infty} \frac{1}{K_{n}(\omega)} \Gamma_{n}^{\star}\left(e^{j \omega}\right) M_{n}(F) Q \widetilde{\Gamma}_{n}\left(e^{j \omega}\right)\right]_{k}=\left[F(\omega) \lim _{n \rightarrow \infty} \frac{1}{K_{n}(\omega)} \widetilde{\Gamma}_{n}^{\star}\left(e^{j \omega}\right) Q \widetilde{\Gamma}_{n}\left(e^{j \omega}\right)\right]_{k}
$$

where

$$
K_{n}(\omega) \triangleq \sum_{k=1}^{n}\left|\mathcal{B}_{k}\left(e^{j \omega}\right)\right|^{2}=\sum_{k=1}^{n} \frac{1-\left|\xi_{k}\right|^{2}}{\left|e^{j \omega}-\xi_{k}\right|^{2}}, \quad \widetilde{\Gamma}_{n}\left(e^{j \omega}\right) \triangleq \Gamma_{n}\left(e^{j \omega}\right) \otimes I_{\ell}
$$


Proof. Define, for a given fixed $\omega \in[-\pi, \pi]$, the function $\widetilde{G}(\sigma)=\widetilde{F}(\sigma)-\widetilde{F}(\omega)$ of $\sigma$. Then $M_{n}(\widetilde{F})=$ $M_{n}(\widetilde{G})+I_{n} \otimes \widetilde{F}(\omega)$ and therefore, by Lemma B.3

$$
\begin{aligned}
\Gamma_{n}^{\star}\left(e^{j \omega}\right) M_{n}(F) Q \widetilde{\Gamma}_{n}\left(e^{j \omega}\right) & =L^{T} \widetilde{\Gamma}_{n}^{\star}\left(e^{j \omega}\right) M_{n}(\widetilde{F}) Q \widetilde{\Gamma}_{n}\left(e^{j \omega}\right) \\
& =L^{T} \widetilde{\Gamma}_{n}^{\star}\left(e^{j \omega}\right) M_{n}(\widetilde{G}) Q \widetilde{\Gamma}_{n}\left(e^{j \omega}\right)+L^{T} \widetilde{\Gamma}_{n}^{\star}\left(e^{j \omega}\right)\left[I_{n} \otimes \widetilde{F}(\omega)\right] Q \widetilde{\Gamma}_{n}\left(e^{j \omega}\right)
\end{aligned}
$$

Considering now the second term in (B.58)

$$
\begin{aligned}
L^{T} \widetilde{\Gamma}_{n}^{\star}\left(e^{j \omega}\right)\left[I_{n} \otimes \widetilde{F}(\omega)\right] Q \widetilde{\Gamma}_{n}\left(e^{j \omega}\right) & =\left[\Gamma_{n}^{\star}\left(e^{j \omega}\right) \otimes L^{T}\right]\left[I_{n} \otimes \widetilde{F}(\omega)\right] Q \widetilde{\Gamma}_{n}\left(e^{j \omega}\right) \\
& =\left[\Gamma_{n}^{\star}\left(e^{j \omega}\right) \otimes\left[L^{T} \widetilde{F}(\omega)\right]\right] Q \widetilde{\Gamma}_{n}\left(e^{j \omega}\right) \\
& =\left[\Gamma_{n}^{\star}\left(e^{j \omega}\right) \otimes F(\omega)\right] Q \widetilde{\Gamma}_{n}\left(e^{j \omega}\right) \\
& =F(\omega) \widetilde{\Gamma}_{n}^{\star}\left(e^{j \omega}\right) Q \widetilde{\Gamma}_{n}\left(e^{j \omega}\right) .
\end{aligned}
$$

Therefore, (B.58) leads to

$$
\begin{aligned}
& \frac{1}{K_{n}(\omega)}\left|\left[\Gamma_{n}^{\star}\left(e^{j \omega}\right) M_{n}(F) Q \widetilde{\Gamma}_{n}\left(e^{j \omega}\right)\right]_{k}-\left[F(\omega) \widetilde{\Gamma}_{n}^{\star}\left(e^{j \omega}\right) Q \widetilde{\Gamma}_{n}\left(e^{j \omega}\right)\right]_{k}\right| \\
= & \frac{1}{K_{n}(\omega)}\left|\left[L^{T} \widetilde{\Gamma}_{n}^{\star}\left(e^{j \omega}\right) M_{n}(\widetilde{G}) Q \widetilde{\Gamma}_{n}\left(e^{j \omega}\right)\right]_{k}\right| \\
\leq & \frac{1}{K_{n}(\omega)} \sum_{p=1}^{\ell}\left|\left[\widetilde{\Gamma}_{n}^{\star}\left(e^{j \omega}\right) M_{n}(\widetilde{G}) Q \widetilde{\Gamma}_{n}\left(e^{j \omega}\right)\right]_{p, k}\right| .
\end{aligned}
$$

However, since the matrix 2-norm is invariant under a similarity transformation

$$
\begin{aligned}
& \frac{1}{K_{n}(\omega)}\left|\left[\widetilde{\Gamma}_{n}^{\star}\left(e^{j \omega}\right) M_{n}(\widetilde{G}) Q \widetilde{\Gamma}_{n}\left(e^{j \omega}\right)\right]_{p, k}\right| \\
= & \frac{1}{K_{n}(\omega)}\left|\left[\widetilde{\Gamma}_{n}^{\star}\left(e^{j \omega}\right) M_{n}^{\frac{1}{2}}(\widetilde{G})\left[M_{n}^{\frac{1}{2}}(\widetilde{G}) Q M_{n}^{-\frac{1}{2}}(\widetilde{G})\right] M_{n}^{\frac{1}{2}}(\widetilde{G}) \widetilde{\Gamma}_{n}\left(e^{j \omega}\right)\right]_{p, k}\right| \\
\leq & \frac{\|Q\|_{2}}{K_{n}(\omega)}\left|\left[\widetilde{\Gamma}_{n}^{\star}\left(e^{j \omega}\right) M_{n}(\widetilde{G}) \widetilde{\Gamma}_{n}\left(e^{j \omega}\right)\right]_{p, k}\right|
\end{aligned}
$$

where the matrix square roots and inverse square roots are guaranteed to exist by virtue of $M_{n}(\widetilde{G})>0$ being guaranteed [19] because the assumed continuity of $\widetilde{F}$ implies $\widetilde{G} \neq 0$ (or else it is identically zero, in which case the Lemma is trivially shown because then $F$ is a constant). Finally, by Lemma B.5

$$
\lim _{n \rightarrow \infty}\left[\frac{\widetilde{\Gamma}_{n}^{\star}\left(e^{j \omega}\right) M_{n}(\widetilde{G}) \widetilde{\Gamma}_{n}\left(e^{j \omega}\right)}{K_{n}(\omega)}\right]_{p, k}=[\widetilde{G}(\omega)]_{p, k}=0 .
$$

Lemma B.5. Suppose $F(\omega) \in \mathbf{C}^{\nu \times \ell}$ is a matrix valued function which is componentwise Lipschitz continuous of some order $\eta>0$ on $[-\pi, \pi]$. Suppose also that in the definition of the $\left\{\mathcal{B}_{k}\right\}$ forming the $\Gamma_{n}$ (and hence $M_{n}(\cdot)$ ) that the poles $\left\{\xi_{k}\right\}$ satisfy $\left|\xi_{k}\right| \leq 1-\delta$ for some $\delta>0$. Then

$$
\lim _{n \rightarrow \infty}\left[\frac{1}{K_{n}(\omega)}\left[\Gamma_{n}^{\star}\left(e^{j \omega}\right) \otimes I_{\ell}\right] M_{n}(F)\left[\Gamma_{n}\left(e^{j \omega}\right) \otimes I_{\ell}\right]\right]_{k, p}=[F(\omega)]_{k, p} .
$$


Proof.

$$
\begin{gathered}
{\left[\frac{\left[\Gamma_{n}^{\star}\left(e^{j \omega}\right) \otimes I_{\ell}\right] M_{n}(F)\left[\Gamma_{n}\left(e^{j \omega}\right) \otimes I_{\ell}\right]}{K_{n}(\omega)}\right]_{k, p}} \\
=\left[\frac{1}{K_{n}(\omega)} \frac{1}{2 \pi} \int_{-\pi}^{\pi}\left[\Gamma_{n}^{\star}\left(e^{j \omega}\right) \otimes I_{\ell}\right]\left[\Gamma_{n}\left(e^{j \sigma}\right) \Gamma_{n}^{\star}\left(e^{j \sigma}\right) \otimes F(\sigma)\right]\left[\Gamma_{n}\left(e^{j \omega}\right) \otimes I_{\ell}\right] \mathrm{d} \sigma\right]_{k, p} \\
=\frac{1}{2 \pi K_{n}(\omega)} \int_{-\pi}^{\pi} \Gamma_{n}^{\star}\left(e^{j \omega}\right) \Gamma_{n}\left(e^{j \sigma}\right) \Gamma_{n}^{\star}\left(e^{j \sigma}\right) \Gamma_{n}\left(e^{j \omega}\right)[F(\sigma)]_{k, p} \mathrm{~d} \sigma \\
=\frac{1}{K_{n}(\omega)} \Gamma_{n}^{\star}\left(e^{j \omega}\right) M_{n}\left([F]_{k, p}\right) \Gamma_{n}\left(e^{j \omega}\right) .
\end{gathered}
$$

This expression converges to $[F(\omega)]_{k, p}$ as $n \rightarrow \infty$ as a consequence of Lemma B.1.

Lemma B.6. Suppose that $F(\omega) \in \mathbf{R}^{\ell \times \nu}$ and $H(\omega) \in \mathbf{R}^{\nu \times \ell}$ are arbitrary, positive, matrix-valued functions which are componentwise Lipschitz continuous functions of order $\eta>0$ for all $\omega \in[-\pi, \pi]$ and that each $\left|\xi_{k}\right|$ in the definition of $M_{n}(\cdot)$ satisfies $\left|\xi_{k}\right| \leq 1-\delta$ for some $\delta>0$. Suppose also that $\beta \in \mathbf{R}^{n}$ and that $\|\beta\|_{1}<\infty$. Then

$$
\lim _{n \rightarrow \infty}\left|\left[\left[\beta^{\star} \otimes I_{\ell}\right]\left[M_{n}(F) M_{n}(H)-M_{n}(F H)\right]\left[\beta \otimes I_{\ell}\right]\right]_{k, p}\right|=0,
$$

where $[\cdot]_{k, p}$ denotes the $k, p^{\prime}$ th entry of a matrix.

Proof. This follows directly from Lemma B.7 on noticing that

$$
\left[M_{n}(F) M_{n}(H)-M_{n}(F H)\right]_{k, p}=\sum_{r=1}^{\nu} M_{n}\left([F]_{k, r}\right) M_{n}\left([H]_{r, p}\right)-\sum_{r=1}^{\nu} M_{n}\left([F]_{k, r}[H]_{r, p}\right)
$$

and that if $\|\beta\|_{1}<\infty$ then $\exists C<\infty, \epsilon>0$ such that $\left|\beta_{k}\right|<\frac{C}{k^{1 / 2+\epsilon}}$.

Lemma B.7. Suppose that $f(\omega)$ and $h(\omega)$ are positive and Lipschitz continuous functions of order $\eta>0$ for all $\omega \in[-\pi, \pi]$, and that each $\xi_{k}$ in the definition of $M_{n}(\cdot)$ satisfies $\left|\xi_{k}\right| \leq \delta<1$ for some $\delta>0$. Then

$$
\left\|M_{n}(f) M_{n}(h)-M_{n}(f h)\right\| \rightarrow 0 \text { as } n \rightarrow \infty,
$$

where the norm $\|\cdot\|$ is defined for any matrix $Q \in \mathbf{C}^{n \times n}$ as $\|Q\| \triangleq \sup _{x \in \Pi} \frac{x^{\star} Q x}{x^{\star} x}$ where the set $\Pi$ is defined for some $C_{1}<\infty$ and some $\varepsilon>0$ as

$$
\Pi \triangleq\left\{x \in \mathbf{C}^{n}:\left|x_{k}\right|<\frac{C_{1}}{k^{1 / 2+\epsilon}}\right\} .
$$

Proof. By Lemma B.8 any $x \in \Pi$ may be written as $x=\Lambda_{n} y$, where $y \in \mathbf{C}^{n}$ and

$$
\Lambda_{n}=\left[\frac{\Gamma_{n}\left(e^{j \omega_{1}}\right)}{\sqrt{K_{n}\left(\omega_{1}\right)}}, \frac{\Gamma_{n}\left(e^{j \omega_{2}}\right)}{\sqrt{K_{n}\left(\omega_{2}\right)}}, \ldots, \frac{\Gamma_{n}\left(e^{j \omega_{n}}\right)}{\sqrt{K_{n}\left(\omega_{n}\right)}}\right]
$$

where the frequencies $\omega_{1}, \omega_{2}, \cdots, \omega_{n}$ may be chosen arbitrarily subject to them all being distinct from one another. Denoting the elements of $y$ as $y_{1}, y_{2}, \cdots, y_{n}$ and using $[\cdot]_{k, \ell}$ to denote the $k, \ell^{\prime} t h$ element of a matrix

$$
\begin{aligned}
\lim _{n \rightarrow \infty}\left|x^{\star}\left[M_{n}(f) M_{n}(h)-M_{n}(f h)\right] x\right| & =\lim _{n \rightarrow \infty}\left|y^{\star} \Lambda_{n}^{\star}\left[M_{n}(f) M_{n}(h)-M_{n}(f h)\right] \Lambda_{n} y\right| \\
& \leq \lim _{n \rightarrow \infty} \sum_{k=1}^{n} \sum_{\ell=1}^{n}\left|\overline{y_{k}} y_{\ell}\right|\left|\left[\Lambda_{n}^{\star}\left[M_{n}(f) M_{n}(h)-M_{n}(f h)\right] \Lambda_{n}\right]_{k, \ell}\right| .
\end{aligned}
$$


Using the Cauchy-Schwarz Inequality and Theorem 5.1 in [19],

$$
\begin{aligned}
& \left|\left[\Lambda_{n}^{\star}\left[M_{n}(f) M_{n}(h)-M_{n}(f h)\right] \Lambda_{n}\right]_{k, \ell}\right|^{2} \\
= & \left|\frac{\Gamma_{n}^{\star}\left(e^{j \omega_{k}}\right)\left[M_{n}(f) M_{n}(h)-M_{n}(f h)\right] \Gamma_{n}\left(e^{j \omega_{\ell}}\right)}{\sqrt{K_{n}\left(\omega_{k}\right)} \sqrt{K_{n}\left(\omega_{\ell}\right)}}\right|^{2} \\
\leq & \frac{\Gamma_{n}^{\star}\left(e^{j \omega_{k}}\right)\left[M_{n}(f) M_{n}(h)-M_{n}(f h)\right]\left[M_{n}(f) M_{n}(h)-M_{n}(f h)\right]^{\star} \Gamma_{n}\left(e^{j \omega_{k}}\right)}{K_{n}\left(\omega_{k}\right)} \\
\leq & M n^{\frac{-\eta}{\eta+2}} \log ^{4} n,
\end{aligned}
$$

for some constant $M$. Therefore,

$$
\sum_{k=1}^{n} \sum_{\ell=1}^{n}\left|\overline{y_{k}} y_{\ell}\right|\left|\left[\Lambda_{n}^{\star}\left[M_{n}(f) M_{n}(h)-M_{n}(f h)\right] \Lambda_{n}\right]_{k, \ell}\right| \leq \sqrt{C n^{\frac{-\eta}{\eta+2}}} \log ^{2} n\left(\sum_{k=1}^{n}\left|y_{k}\right|\right)^{2} .
$$

Furthermore

$$
\left|x_{k}\right| \geq \min _{1 \leq \ell \leq n}\left|\left[\Lambda_{n}\right]_{k, \ell}\right| \sum_{\ell=1}^{n}\left|y_{\ell}\right|, \quad \forall k \in[1, n]
$$

Therefore, via the assumption that $\exists C$ such that $\left|x_{k}\right| \leq \frac{C}{n^{1 / 2+\epsilon}}$ for some $\epsilon>0$, and also by the assumption of $\left|\xi_{k}\right| \leq 1-\delta$ for some $\delta>0$, so that by Lemma 4.2 of [19] $\left|\left[\Lambda_{n}\right]_{k, \ell}\right| \geq \delta / 2$, there exists a $C^{\prime}>0$ such that

$$
\frac{C}{n^{1 / 2+\epsilon}} \geq \min _{1 \leq \ell \leq n}\left|\left[\Lambda_{n}\right]_{k, \ell}\right| \sum_{\ell=1}^{n}\left|y_{\ell}\right| \geq \frac{C^{\prime}}{\sqrt{n}} \sum_{\ell=1}^{n}\left|y_{\ell}\right| .
$$

Therefore $\sum_{\ell=1}^{n}\left|y_{\ell}\right| \leq \frac{C^{\prime \prime}}{n^{\epsilon}}$ for some $C^{\prime \prime}<\infty$ so that for some $C^{\prime \prime \prime}<\infty$,

$$
\left\|M_{n}(f) M_{n}(h)-M_{n}(f h)\right\| \leq \frac{C^{\prime \prime \prime} \log ^{2} n}{n^{\epsilon} n^{\eta /(2 \eta+4)}} \rightarrow 0 \text { as } n \rightarrow \infty
$$

Lemma B.8. The vectors $\left\{\Gamma_{n}\left(e^{j \omega_{k}}\right): 1 \leq k \leq n, \omega_{k} \in[-\pi, \pi], \omega_{\ell} \neq \omega_{k}\right\}$ span $\mathbf{C}^{n}$.

Proof. Suppose not. Then $\exists x \in \mathbf{C}^{n}, x \neq 0$ such that

$$
x^{T} \Gamma_{n}\left(e^{j \omega_{k}}\right)=0 \quad \text { for all } 1 \leq k \leq n .
$$

However, denoting the elements of $x$ as $x_{1}, x_{2}, \ldots, x_{n}$ then $x^{T} \Gamma_{n}(z)=\sum_{k=1}^{n} x_{k} \mathcal{B}_{k}(z)$ which, by construction, is an $n^{\text {th }}$-order rational function of $z$ of relative degree 1 , therefore possessing $n-1$ zeros. Hence, $x^{T} \Gamma_{n}(z)$ can be zero for at most $n-1$ distinct values of $z$, and hence (B.59) cannot be satisfied for $n$ distinct values of $\left\{\omega_{k}\right\}$.

Theorem B.9. If $X$ and $Y$ are independent random variables and $f$ and $g$ are Borel measurable functions, then $f(X)$ and $g(Y)$ are independent random variables. 
Proof. Let $B_{\ell}, B_{m} \in \mathcal{B}^{1}$, where $\mathcal{B}^{1}$ is the Euclidean Borel field on $\mathbf{R}^{1}$, and let $\mathbf{P}$ be the relevant probability measure. Then

$$
\begin{aligned}
\mathbf{P}\left\{\left(f(X) \in \mathcal{B}_{\ell}\right) \cap\left(g(Y) \in \mathcal{B}_{m}\right)\right\} & =\mathbf{P}\left\{\left(X \in f^{-1}\left(\mathcal{B}_{\ell}\right)\right) \cap\left(Y \in g^{-1}\left(\mathcal{B}_{m}\right)\right)\right\} \\
& =\mathbf{P}\left\{X \in f^{-1}\left(\mathcal{B}_{\ell}\right)\right\} . \mathbf{P}\left\{Y \in g^{-1}\left(\mathcal{B}_{m}\right)\right\} \\
& =\mathbf{P}\left\{f(X) \in \mathcal{B}_{\ell}\right\} . \mathbf{P}\left\{g(Y) \in \mathcal{B}_{m}\right\} .
\end{aligned}
$$

Since this is true for every choice of $B_{\ell}$ and $B_{m}, f(X)$ and $g(Y)$ are independent.

\section{References}

[1] Special issue on system identification for robust control design, IEEE Transactions on Automatic Control, 37 (1992).

[2] K. Chung, A Course in Probability Theory, Harcourt, Brace and World Inc., 1968.

[3] G. Goodwin And R. Payne, Dynamic System Identification, Academic Press, 1977.

[4] G. Goodwin And K. Sin, Adaptive Filtering Prediction and Control, Prentice-Hall, Inc., New Jersey, 1984.

[5] L. GuO AND L. LuUng, The role of model validation for assessing the size of the unmodelled dynamics, in Proc. 33rd IEEE Conference on Decision and Control, Lake Buena Vista, FL, Dec 1994, pp. 3894-3899.

[6] E. Hannan AND D. Nicholls, The estimation of the prediction error variance, Journal of the American Statistical Association, 72 (1977), pp. 834-840.

[7] P. Heuberger, P.M.J. VAn den Hof, And O. Bosgra, A generalized orthonormal basis for linear dynamical systems, IEEE Transactions on Automatic Control, AC-40 (1995), pp. 451465.

[8] R. Jennrich, Asymptotic properties of non-linear least squares estimators, The Annals of Mathematical Statistics, 40 (1969), pp. 633-643.

[9] R. Koust, M. LAU, And S. Boyd, Set-membership identification of systems with parametric and nonparametric uncertainty, IEEE Transactions on Automatic Control, AC-37 (1992), pp. 929-941.

[10] L. LJUng, System Identification: Theory for the User, (2nd edition), Prentice-Hall, Inc., New Jersey, 1999.

[11] L.LJUnG, Convergence analysis of parametric identification methods, IEEE Transactions on Automatic Control, AC-23 (1978), pp. 770-783.

[12] — Asymptotic variance expressions for identified black-box transfer function models, IEEE Transactions on Automatic Control, AC-30 (1985), pp. 834-844.

[13] L.LJUng AND P.E.CAINES, Asymptotic Normality of prediction error estimators for approximate system models, Stochastics, 3 (1979), pp. 29-46.

[14] L.LuUng AND Z.D.YuAn, Asymptotic properties of black-box identification of transfer functions, IEEE Transactions on Automatic Control, 30 (1985), pp. 514-530. 
[15] M. Milanese And A. Vicino, Optimal estimation theory for dynamic systems with set membership uncertainty:An overview, Automatica, 27 (1991), pp. 997-1009.

[16] K. NARENDRA AND P. Gallman, In iterative method for the identification of nonlinear systems using a Hammerstein model, IEEE Transactions on Automatic Control, 11 (1966), pp. 546550 .

[17] B. Ninness And G. Goodwin, Estimation of model quality, Automatica, 31 (1995), pp. 3274.

[18] B. Ninness, H. Hualmarsson, And F. Gustafsson, The fundamental role of general orthonormal bases in system identification, IEEE Transactions on Automatic Control, 44 (1999), pp. 1384-1406.

[19] — Generalised Fourier and Toeplitz results for rational orthonormal bases, SIAM Journal on Control and Optimization, 37 (1999), pp. 429-460.

[20] P.M.J. VAn Den Hof, P.S.C. Heuberger, And J. Bokor, System identification with generalized orthonormal basis functions, Automatica, 31 (1995), pp. 1821-1834.

[21] B. Pötscher And I. Prucha, Dynamic Nonlinear Econometrics Models, Springer-Verlag, Berlin-Heidelberg, 1997.

[22] R. PRICE, A useful theorem for nonlinear devices having gaussian inputs, IRE Transactions on Information Theory, 4 (1958), pp. 69-72.

[23] S. Rangan, G. Wolodkin, And K. Poolla, New results for Hammerstein system identification, in Proceedings of the 34th IEEE Conference on Decision and Control, 1995, pp. 697-702.

[24] R. Smith And M. DAhleh, The Modelling of Uncertainty in Control Systems, Springer Verlag, 1994.

[25] G. TAo And P. V. Koкотović, Adaptive Control of Systems with Actuator and Sensor Nonlinearities, Adaptive and Learning Systems for Signal Processing, Communications and Control, John Wiley and Sons, 1996.

[26] B. WAhlberg, System identification using Laguerre models, IEEE Transactions on Automatic Control, AC-36 (1991), pp. 551-562.

[27] B. Wahlberg, System identification using Kautz models, IEEE Transactions on Automatic Control, AC-39 (1994), pp. 1276-1282.

[28] B. WAHLBERG AND L. LJUNG, Hard frequency-domain model error bounds from least-squares like identification techniques, IEEE Transactions on Automatic Control, 37 (1992), pp. 900-912.

[29] A. Weinmann, Uncertain Models and Robust Control, Springer-Verlag, New York, 1991. 